\title{
General Poincaré gauge theory: Hamiltonian structure and particle spectrum
}

\author{
M. Blagojević and B. Cvetković* \\ Institute of Physics, University of Belgrade \\ Pregrevica 118, 11080 Belgrade, Serbia
}

July 20, 2018

\begin{abstract}
Basic aspects of the Hamiltonian structure of the parity-violating Poincaré gauge theory are studied. We found all possible primary constraints, identified the corresponding critical parameters, and constructed the generic form of the canonical Hamiltonian. In addition to being important in their own right, these results offer dynamical information that is essential for a proper understanding of the particle spectrum of the theory, calculated in the weak field approximation around the Minkowski background.
\end{abstract}

\section{Introduction}

Weyl's idea of gauge invariance [1] turned out to be a key principle underlying the dynamical structure of all the fundamental physical interactions. Following this idea and the subsequent works of Yang, Mills and Utiyama 2], Kibble and Sciama 3] formulated a new theory of gravity, the Poincaré gauge theory (PG, aka PGT), based on gauging the Poincaré group of spacetime symmetries. In PG, spacetime is characterized by a Riemann-Cartan geometry, in which the torsion and curvature are the field strengths associated with the translation and Lorentz subgroups of the Poincaré group; for more details, see [4, 5, 6, 7, 8, 9, 10,

Earlier investigations of PG were mostly focused on the class of parity preserving Lagrangians quadratic in the field strengths; see, for instance, Hayashi and Shirafuji [5], or Obukhov [11. We denote this class of models as $\mathrm{PG}^{+}$. Sezgin and Niuwenhuizen [12] analyzed the particle spectrum of $\mathrm{PG}^{+}$in the weak field approximation around the Minkowski background $M_{4}$. Using the absence of ghosts and tachyons as physical requirements, they found a number of restrictions on the $\mathrm{PG}^{+}$parameters that ensure the propagating torsion modes to be well behaved.

General dynamical aspects of $\mathrm{PG}^{+}$, including the identification of its physical degrees of freedom, are most naturally understood in Dirac's Hamiltonian approach for constrained dynamical systems [13. Blagojević and Nikolić [14, 15] started a systematic Hamiltonian analysis of $\mathrm{PG}^{+}$, focusing on its generic aspects. They identified a subset of the primary

\footnotetext{
*Email addresses: mb@ipb.ac.rs, cbranislav@ipb.ac.rs
} 
constraints that are always present ("sure" constraints, associated to the local Poincaré symmetry). Moreover, if certain critical parameters vanished, they found additional primary constraints ("if-constraints"), constructed the total Hamiltonian, and discussed certain aspects of the consistency procedure. Further advances in this direction were made by Cheng et al. [16] and Chen et al [17], who found that the nonlinear nature of constraints may drastically change the number of propagating modes obtained in the linearized analysis. Yo and Nester [18] made a detailed study of this phenomenon in $\mathrm{PG}^{+}$, concluding that there are apparently only two good propagating torsion modes. For an interesting application of this result to cosmology, see Shie et al. [19].

There are no physical arguments that favor the conservation of parity in the gravitational interaction. Parity violating models based on the general PG, with all possible quadratic invariants in the Lagrangian, were considered already in the 1980s [20], but the subject remained without wider response. Recently, there has been increased interest in a better understanding of both the basic structure and various dynamical aspects of these models, including cosmological applications and wave solutions [21, 22, 23, 24, 25, 26, 27]. In particular, one should mention the analysis of the particle spectrum carried out by Karananas 25], who made a suitable extension of the weak field approximation method used earlier in $\mathrm{PG}^{+}[12$ and applied it to the general PG. According to his results, it seems that the set of good modes that can coexist is significantly enlarged in comparison to $\mathrm{PG}^{+}$.

The objective of the present work is to examine the Hamiltonian structure of the general PG, based on the if-constraint formalism [14, 15, 18, and use it to clarify the physical content of its particle spectrum, calculated in the weak field approximation around $M_{4}$. In this regard, a particularly important role is played by both the critical parameters appearing in the analysis of the primary constraints, and the structure of the canonical Hamiltonian. By comparing the properties of the particle spectrum to those found in Ref. [25], we noted certain differences. On the other side, elements of the Hamiltonian structure developed here can be a good starting point for studying the nonlinear dynamics of PG.

The paper is organized as follows. In Sec. 2, we give a short account of the Lagrangian formalism for the general PG. In Secs. 3 and 4, we find the canonical critical parameters, identify the related if-constraints, and construct the generic, "most dynamical" canonical Hamiltonian, determined by the nonvanishing critical parameters. Then, in Secs. 5 and 6. we derive the linearized gravitational field equations and use them to identify the mass eigenvalues of the torsion modes. The conditions for the absence of ghosts and tachyons, as well as the reality conditions of the mass eigenvalues, are examined in Sec. 7. Essential features of the particle spectrum are either tested by, or derived from the Hamiltonian structure of PG. In contrast to the results obtained in [25], we show that the two spin-2 torsion modes cannot propagate simultaneously. In Sec. 8, we give a short summary of our results, and six appendices contain useful technical details, including an outline of the Hamiltonian formalism describing the case of vanishing critical parameters.

Our conventions are as follows. The Latin indices $(i, j, \ldots)$ are the local Lorentz indices, the Greek indices $(\mu, \nu, \ldots)$ are the coordinate indices, and both run over $0,1,2,3$; the orthonormal frame (tetrad) is $b_{\mu}^{i}$, the inverse tetrad is $h_{i}{ }^{\mu}$, the Lorentz connection is $\omega^{i j}{ }_{\mu}$, $\eta_{i j}=(1,-1,-1,-1)$ and $g_{\mu \nu}=\eta_{i j} b_{\mu}^{i} b^{j}{ }_{\nu}$ are the metric components in the local Lorentz and coordinate frame, respectively; a totally antisymmetric tensor $\varepsilon_{i j k l}$ is normalized to $\varepsilon_{0123}=+1$, and the dual of an antisymmetric tensor $X_{i j}$ is ${ }^{\star} X_{i j}=(1 / 2) \varepsilon_{i j}{ }^{m n} X_{m n}$. 


\section{Lagrangian formalism}

In this section, we give a short account of the Lagrangian formalism for the general parity violating PG. Basic dynamical variables are the tetrad field $b^{i}=b^{i}{ }_{\mu} d x^{\mu}$ and the antisymmetric spin connection $\omega^{i j}=\omega^{i j}{ }_{\mu} d x^{\mu}=-\omega^{j i}$ (1-forms), which represent the gauge potentials associated with translations and Lorentz transformations, respectively. The corresponding field strengths are the torsion and the curvature (2-forms),

$$
\begin{aligned}
& T^{i}:=d b^{i}+\omega^{i}{ }_{k} \wedge b^{k}=\frac{1}{2} T^{i}{ }_{\mu \nu} d x^{\mu} \wedge d x^{\nu}, \\
& R^{i j}:=d \omega^{i j}+\omega^{i}{ }_{k} \wedge \omega^{k j}=\frac{1}{2} R^{i j}{ }_{\mu \nu} d x^{\mu} \wedge d x^{\nu},
\end{aligned}
$$

which satisfy the Bianchi identities

$$
\nabla T^{i}=R_{k}^{i} \wedge b^{k}, \quad \nabla R^{i j}=0 .
$$

The underlying spacetime continuum is described by Riemann-Cartan geometry [7, 8, 9].

\subsection{Field equations}

The PG dynamics is determined by a Lagrangian $L=L_{M}+L_{G}$, where $L_{M}$ describes matter and its interaction with gravity, and $L_{G}$ is the pure gravitational part. In the framework of tensor calculus, the gravitational field equations in vacuum are obtained by varying the action $I_{G}=\int d^{4} x L_{G}\left(b^{i}, T_{i j k}, R_{i j k l}\right)$ with respect to $b_{\mu}^{i}$ and $\omega^{i j}{ }_{\mu}$. After introducing the covariant gravitational momenta

$$
H_{i}{ }^{\mu \nu}:=\frac{\partial L_{G}}{\partial T^{i}{ }_{\mu \nu}}, \quad H_{i j}{ }^{\mu \nu}:=\frac{\partial L_{G}}{\partial R^{i j}{ }_{\mu \nu}},
$$

and the associated energy-momentum and spin currents

$$
E_{i}^{\nu}:=\frac{\partial L_{G}}{\partial b^{i}{ }_{\mu}}, \quad E_{i j}{ }^{\mu}:=\frac{\partial L_{G}}{\partial \omega^{i j}{ }_{\mu}},
$$

the gravitational field equations take a compact form

$$
\begin{aligned}
& \text { (1ST) } \quad \mathcal{E}_{i}{ }^{\nu}:=-\frac{\delta L_{G}}{\delta b^{i}{ }_{\mu}}=\nabla_{\mu} H_{i}{ }^{\mu \nu}-E_{i}{ }^{\nu}=0, \\
& (2 \mathrm{ND}) \quad \mathcal{E}_{i j}{ }^{\nu}:=-\frac{\delta L_{G}}{\delta \omega^{i j}{ }_{\mu}}=\nabla_{\mu} H_{i j}{ }^{\mu \nu}-E_{i j}{ }^{\nu}=0 .
\end{aligned}
$$

The explicit expressions for the energy-momentum and spin currents are given by

$$
\begin{aligned}
& E_{i}{ }^{\nu}=h_{i}{ }^{\nu} L_{G}-T_{k i}^{m} H_{m}{ }^{k \nu}-\frac{1}{2} R_{k i}^{m n} H_{m n}{ }^{k \nu}, \\
& E_{i j}{ }^{\mu}=-2 H_{[i j]}{ }^{\mu} .
\end{aligned}
$$

In the presence of matter, the right-hand sides of (2.4a $)$ and $(2.4 \mathrm{~b})$ contain the corresponding matter currents. 


\subsection{Quadratic PG models}

We assume the Lagrangian density $L_{G}$ to contain all possible quadratic invariants, constructed out of the three irreducible components of the torsion, and the six irreducible components of the curvature (Appendix A) . Relying on the Lagrangian 4-form given in Ref. [27], one finds that the corresponding Lagrangian density has the form $L_{G}=b \mathcal{L}_{G}$, where $b:=\operatorname{det}\left(b_{\mu}^{i}\right)$ and

$$
\begin{aligned}
\mathcal{L}_{G}= & -\left(a_{0} R+2 \Lambda_{0}\right)-\bar{a}_{0} X \\
& +\frac{1}{2} T^{i j k} \sum_{n=1}^{3}\left(a_{n}{ }^{(n)} T_{i j k}-\bar{a}_{n}{ }^{\star}{ }^{(n)} T_{i j k}\right), \\
& +\frac{1}{4} R^{i j k l} \sum_{n=1}^{6}\left(b_{n}{ }^{(n)} R_{i j k l}-\bar{b}_{n}{ }^{\star}{ }^{(n)} R_{i j k l}\right) .
\end{aligned}
$$

Here, the irreducible components of the field strengths are defined in Appendix A, the parity even and parity odd sectors are described by the parameters $\left(a_{n}, b_{n}, \Lambda_{0}\right)$ and $\left(\bar{a}_{n}, \bar{b}_{n}\right)$, respectively, and the star symbol denotes the duality operation with respect to the frame indices of the field strengths. Another form of $\mathcal{L}_{G}$, useful for comparison with the literature, is given in Appendix $\mathrm{B}$. Knowing $\mathcal{L}_{G}$, one finds that the covariant momentum densities (2.3a) can be written in the form $H_{i m n}=b \mathcal{H}_{i m n}$ and $H_{i j m n}=b \mathcal{H}_{i j m n}$, where

$$
\begin{aligned}
& \mathcal{H}_{i m n}=2 \sum_{n=1}^{3}\left(a_{n}{ }^{(n)} T_{i m n}-\bar{a}_{n}{ }^{\star} T_{i m n}\right), \\
& \mathcal{H}_{i j m n}={ }^{L} \mathcal{H}_{i j m n}+\mathcal{H}_{i j m n}^{\prime},
\end{aligned}
$$

and

$$
\begin{gathered}
{ }^{L} \mathcal{H}_{i j m n}=-2 a_{0}\left(\eta_{i m} \eta_{j n}-\eta_{j m} \eta_{i n}\right)+2 \bar{a}_{0} \varepsilon_{i j m n} \\
\mathcal{H}_{\text {ijmn }}^{\prime}=2 \sum_{n=1}^{6}\left(b_{n}{ }^{(n)} R_{i j m n}-\bar{b}_{n}{ }^{\star}{ }^{(n)} R_{i j m n}\right) .
\end{gathered}
$$

\subsection{On the choice of Lagrangian parameters}

In the Lagrangian (2.6), the two parity sectors are presented in a very symmetric way, but the set of three identities (A.3a) implies that not all of the parameters $\left(\bar{a}_{n}, \bar{b}_{n}\right)$ are independent. To resolve this issue, we choose the conditions

$$
\bar{a}_{2}=\bar{a}_{3}, \quad \bar{b}_{2}=\bar{b}_{4}, \quad \bar{b}_{3}=\bar{b}_{6},
$$

which reduce the number of Lagrangian parameters to $21-3=18$. Note that the above conditions are not unique.

Further freedom in the choice of parameters follows from the existence of three topological invariants. The Euler and Pontryagin invariants are defined by the 4-forms

$$
I_{E}:=R^{i j} \wedge R^{m n} \varepsilon_{m n i j}, \quad I_{P}:=R^{i j} \wedge R_{i j}
$$


respectively, whereas the third invariant is based on the Nieh-Yan identity,

$$
I_{N Y}:=T^{i} \wedge T_{i}-R_{i j} \wedge b^{i} \wedge b^{j} \equiv d\left(b^{i} \wedge T_{i}\right) .
$$

These invariants produce vanishing contributions to the field equations, which implies that not all of the Lagrangian parameters are dynamically independent. Indeed, they can be used to eliminate three more terms from the Lagrangian, leaving us with the final number of $18-3=15$ independent parameters; see Ref. $[23]_{3}$ for more details. In this paper, we use only the conditions (2.8), allowing thereby for an easier comparison to the literature.

For a clear understanding of the physical content of PG, it is convenient to use dimensionless parameters (coupling constants). The Lagrangian parameters in (2.6) are not dimensionless, but the transition to their dimensionless counterparts can be easily realized by suitable rescalings; see for instance Ref. [27]. However, to make the general exposition simpler and more compact, we find it useful to keep the Lagrangian parameters in the form (2.6), which corresponds to using the units $c=\hbar=2 \kappa=1$. The true dimensionless parameters can be reintroduced later whenever needed.

\section{Primary constraints}

Hamiltonian structure is by itself a particularly important aspect of PG as a gauge theory [13. Moreover, it also offers dynamical information that is essential for a proper understanding of the particle spectrum of PG.

We begin the subject by analyzing the primary constraints (PC) of PG. The canonical momenta $\left(\pi_{i}{ }^{\mu}, \Pi_{i j}{ }^{\mu}\right)$ associated to the basic Lagrangian variables $\left(b^{i}{ }_{\mu}, \omega^{i j}{ }_{\mu}\right)$ are

$$
\pi_{i}^{\mu}:=\frac{\partial L_{G}}{\partial\left(\partial_{0} b^{i}{ }_{\mu}\right)}=b \mathcal{H}_{i}{ }^{0 \mu}, \quad \Pi_{i j}{ }^{\mu}:=\frac{\partial L_{G}}{\partial\left(\partial_{0} \omega^{i j}{ }_{\mu}\right)}=b \mathcal{H}_{i j}{ }^{0 \mu}
$$

Since the field strengths do not depend on the velocities $\partial_{0} b_{0}^{i}$ and $\partial_{0} \omega^{i j}{ }_{0}$, the above relations define 10 constraints that are always present in the theory ("sure" PCs), regardless of the values of the coupling constants. They read

$$
\pi_{i}^{0} \approx 0, \quad \Pi_{i j}{ }^{0} \approx 0
$$

and their existence is directly related to the local Poincaré symmetry of PG.

Before we proceed, let us note that at each point of a spatial hypersurface $\Sigma_{0}: x^{0}=$ const., one can define the unit timelike vector $\boldsymbol{n}$, normal to $\Sigma_{0}$. Then, any spacetime vector $V_{k}$ can be decomposed into a component $V_{\perp}$ along $\boldsymbol{n}$, and a component $V_{\bar{k}}$ in the tangent space of ("parallel" to) $\Sigma_{0}$; that is $V_{k}=n_{k} V_{\perp}+V_{\bar{k}}$, where $V_{\perp}=n^{k} V_{k}$ and $n^{k} V_{\bar{k}}=0$ (Appendix C).

To find additional constraints that may appear in (3.1), it is useful to define the parallel gravitational momenta

$$
\begin{aligned}
& \hat{\pi}_{i \bar{k}}:=\pi_{i}{ }^{\alpha} b_{k \alpha}=J \mathcal{H}_{i \perp \bar{k}}, \\
& \hat{\Pi}_{i j \bar{k}}:=\Pi_{i j}{ }^{\alpha} b_{k \alpha}=J \mathcal{H}_{i j \perp \bar{k}},
\end{aligned}
$$

such that $\hat{\pi}_{i \bar{k}} n^{k}=0, \Pi_{i j k} n^{k}=0$, and $J$ is defined by $b=N J$, with $N=n_{k} b_{0}^{k}$. Depending on the values of the coupling constants, these relations may produce additional constraints 
(primary "if-constraints"). In analogy to the above orthogonal-parallel decomposition of a vector $V_{k}$, one can introduce a similar decomposition of the field strengths,

$$
\begin{aligned}
& T_{i k m}=T_{i \bar{k} \bar{m}}+\left(n_{k} T_{i \perp \bar{m}}+n_{m} T_{i \bar{k} \perp}\right)=\bar{T}_{i k m}+\mathcal{T}_{i k m} \\
& R_{i j k m}=R_{i j \bar{k} \bar{m}}+\left(n_{k} R_{i j \perp \bar{m}}+n_{m} R_{i j \bar{k} \perp}\right)=\bar{R}_{i j k m}+\mathcal{R}_{i j k m} .
\end{aligned}
$$

It is very useful for further analysis to know that the parallel components $\bar{T}_{i k m}:=T_{i \bar{k} \bar{m}}$ and $\bar{R}_{i j k m}:=R_{i j \bar{k} \bar{m}}$ are independent not only of the "velocities" $T_{i \perp \bar{m}}, R_{i j \perp \bar{m}}$, but also of the unphysical variables $\left(b_{0}^{i}, \omega^{i j}{ }_{0}\right)$; for more details, see Refs. [7, 14, 15].

\subsection{Torsion sector}

The torsion piece of the Lagrangian (2.6) depends on the velocities $\partial_{0} b^{i}$ only through $T_{i \perp \bar{k}}$. The linearity of $\mathcal{H}_{i \perp \bar{k}}$ in $\bar{T}$ and $\mathcal{T}$ allows us to rewrite (3.3a) in the form

$$
\phi_{i \bar{k}}:=\frac{\hat{\pi}_{i \bar{k}}}{J}-\mathcal{H}_{i \perp \bar{k}}(\bar{T})=\mathcal{H}_{i \perp \bar{k}}(\mathcal{T})
$$

where all possible velocity terms are moved to the right-hand side. Now, we decompose this equation into irreducible parts with respect to the group of rotations in $\Sigma_{0}$ (Appendix C):

$$
\begin{aligned}
& S_{\phi}:=\frac{\hat{\pi}_{\bar{k}}^{\bar{k}}}{J}+\bar{a}_{2} \varepsilon^{\bar{k} \bar{m} \bar{m}} T_{\bar{k} \bar{m} \bar{n}}=-2 a_{2} T_{\bar{k} \perp}^{\bar{k}}, \\
& \phi_{\perp \bar{k}}:=\frac{\hat{\pi}_{\perp \bar{k}}}{J}+\frac{2}{3}\left(a_{1}-a_{2}\right) T_{\bar{m} \bar{k}}^{\bar{m}}+\frac{1}{3}\left(2 \bar{a}_{1}+\bar{a}_{2}\right) \varepsilon_{\bar{k}}{ }^{\bar{m} \bar{n}} T_{\perp \bar{m} \bar{n}} \\
& =\frac{2}{3}\left(2 a_{1}+a_{2}\right) T_{\perp \perp \bar{k}}+\frac{2}{3}\left(\bar{a}_{1}-\bar{a}_{2}\right) \varepsilon_{\bar{k}}^{\bar{m}}{ }^{\bar{n}} T_{\bar{m} \bar{n} \perp}, \\
& { }^{A} \phi_{\bar{\imath} \bar{k}}:=\frac{{ }^{A} \hat{\pi}_{\bar{\imath} \bar{k}}}{J}-\frac{2}{3}\left(a_{1}-a_{3}\right) T_{\perp \bar{\imath} \bar{k}}-\frac{1}{3}\left(\bar{a}_{1}+2 \bar{a}_{3}\right) \varepsilon_{\bar{\imath} \bar{k}}{ }^{\bar{n}} T^{\bar{m} \bar{m} \bar{n}} \\
& =-\frac{2}{3}\left(a_{1}+2 a_{3}\right) T_{[\bar{\imath} \bar{k}] \perp}-\frac{2}{3}\left(\bar{a}_{1}-\bar{a}_{2}\right) \varepsilon_{\bar{\imath} \bar{k}}{ }^{\bar{n}} T_{\perp \perp \bar{n}}, \\
& { }^{T} \phi_{\bar{\imath} \bar{k}}:=\frac{T^{T} \hat{\pi}_{\bar{\imath} \bar{k}}}{J}+\bar{a}_{1}\left[\varepsilon_{(\bar{\imath}}{ }^{\bar{m} \bar{n}} T_{\bar{k}) \bar{m} \bar{n}}-\frac{1}{3} \eta_{\bar{\imath} \bar{k}} \varepsilon^{\bar{k} \bar{m} \bar{n}} T_{\bar{k} \bar{m} \bar{n}}\right]=-2 a_{1}{ }^{T} T_{\bar{\imath} \bar{k} \perp} .
\end{aligned}
$$

Here, the set $\left({ }^{S} \phi, \phi_{\perp \bar{k}},{ }^{A} \phi_{\bar{\imath} \bar{k}},{ }^{T} \phi_{\bar{\imath} \bar{k}}\right)$, defined by the scalar, vector, antisymmetric and tracelesssymmetric parts of $\phi_{i \bar{k}}$, represents the set of all possible new constraints. The mechanism by which these if-constraints become true constraints is simply explained in the parity even case, characterized by four critical parameters: $a_{2},\left(2 a_{1}+a_{2}\right),\left(a_{1}+2 a_{3}\right)$, and $a_{1}$. When some of these parameters vanish, the corresponding velocity terms on the right-hand sides of (3.6) also vanish, and consequently, the associated if-constraints become true constraints. However, if none of the critical parameters vanishes, there are no new constraints.

The same mechanism works also in the general PG. Whereas the critical parameters for ${ }^{S} \phi$ and ${ }^{T} \phi_{\bar{\imath} \bar{k}}$ remain the same as in $\mathrm{PG}^{+}, a_{2}$ and $a_{1}$, the structure of the if-constraints $\phi_{\perp \bar{k}}$ and ${ }^{A} \phi_{\bar{\imath} \bar{k}}$ is more complicated, as the right-hand sides of (3.6b) and (3.6c) depend on two velocities, $T_{[\bar{m} \bar{n}] \perp}$ and $T_{\perp \perp \bar{k}}$. To find the related critical parameters, we first transform ${ }^{A} \phi_{\bar{\imath} \bar{k}}$ into the axial 3-vector ${ }^{A} \phi_{\bar{k}}:=\varepsilon_{\bar{k}}{ }^{\bar{m}} \bar{n} A \phi_{\bar{m} \bar{n}}$, so that (3.6c) goes over into

$$
{ }^{A} \phi_{\bar{k}}=\frac{4}{3}\left(\bar{a}_{1}-\bar{a}_{2}\right) T_{\perp \perp \bar{k}}-\frac{2}{3}\left(a_{1}+2 a_{3}\right) \varepsilon_{\bar{k}}{ }^{\bar{m}} \bar{n} T_{\bar{m} \bar{n} \perp} .
$$


Then, the set of equations involving $\left(\phi_{\perp \bar{k}},{ }^{A} \phi_{\bar{k}}\right)$ can be written in the matrix form as

$$
\left(\begin{array}{c}
\phi_{\perp \bar{k}} \\
{ }^{A} \phi_{\bar{k}}
\end{array}\right)=\frac{2}{3} A\left(\begin{array}{c}
T_{\perp \perp \bar{k}} \\
\varepsilon_{\bar{k}}^{\bar{m} \bar{n}} T_{\bar{m} \bar{n} \perp}
\end{array}\right),
$$

where

$$
\begin{aligned}
& A:=\left(\begin{array}{cc}
2 a_{1}+a_{2} & \bar{a}_{1}-\bar{a}_{2} \\
2\left(\bar{a}_{1}-\bar{a}_{2}\right) & -\left(a_{1}+2 a_{3}\right)
\end{array}\right), \\
& \operatorname{det} A=-\left[\left(2 a_{1}+a_{2}\right)\left(a_{1}+2 a_{3}\right)+2\left(\bar{a}_{1}-\bar{a}_{2}\right)^{2}\right] .
\end{aligned}
$$

If the matrix $A$ has two distinct eigenvalues, one can construct the invertible matrix $P$ that transforms $A$ into a diagonal form, $D_{A}:=P^{-1} A P$. Then, Eq. (3.8a) implies

$$
\phi_{\bar{k}}:=P^{-1}\left(\begin{array}{c}
\phi_{\perp \bar{k}} \\
{ }_{\phi_{\bar{k}}}
\end{array}\right)=\frac{2}{3} D_{A} P^{-1}\left(\begin{array}{c}
T_{\perp \perp \bar{k}} \\
\varepsilon_{\bar{k}}^{\bar{m} \bar{n}} T_{\bar{m} \bar{n} \perp}
\end{array}\right),
$$

where the column $\phi_{\bar{k}}$ represents two diagonalized if-constraints, and the diagonal elements of $D_{A}$ are the critical parameters,

$$
c_{ \pm}(A)=\frac{1}{2}\left(\operatorname{tr} A \pm \sqrt{(\operatorname{tr} A)^{2}-4 \operatorname{det} A}\right) .
$$

More details on this construction can be found in Appendix D, In general, the number of true constraints in (3.9) is equal to the number of vanishing critical parameters.

- The critical parameters of the torsion sector are $a_{2}, c_{ \pm}(A)$, and $a_{1}$.

\subsection{Curvature sector}

For the curvature sector, we use the linearity of $\mathcal{H}_{i j \perp k}^{\prime}$ in $\bar{R}$ and $\mathcal{R}$ to rewrite (3.3b) in the form

$$
\Phi_{i j \bar{k}}:=\frac{\Pi_{i j \bar{k}}}{J}-\mathcal{H}_{i j \perp \bar{k}}(\bar{R})=\mathcal{H}_{i j \perp \bar{k}}^{\prime}(\mathcal{R}) .
$$

The content of the object $\Phi_{i j \bar{k}}$ is described by two three-dimensional (3d) tensors, $\Phi_{i j \bar{k}}=$ $\left(\Phi_{\perp \bar{\jmath} \bar{k}}, \Phi_{\bar{\imath} \bar{\jmath} \bar{k}}\right)$. The irreducible decomposition of $\Phi_{\perp \bar{\jmath} \bar{k}}$ takes the form defined in (‥4):

$$
\begin{aligned}
S_{\Phi} & \equiv \frac{\Pi_{\perp \bar{k}} \bar{k}}{J}+6 a_{0}+\frac{1}{2}\left(b_{4}-b_{6}\right) \underline{R}+\frac{1}{2}\left(\bar{b}_{2}+\bar{b}_{3}\right) \varepsilon^{\bar{k} \bar{m} \bar{n}} R_{\perp \bar{k} \bar{m} \bar{n}} \\
& =\left(b_{4}+b_{6}\right) R_{\perp \perp}-\frac{1}{2}\left(\bar{b}_{2}-\bar{b}_{3}\right) \varepsilon^{\bar{k} \bar{m} \bar{n}} R_{\bar{k} \bar{m} \bar{n} \perp}, \\
{ }^{A} \Phi_{\perp \bar{\jmath} \bar{k}} & \equiv \frac{{ }^{A} \Pi_{\perp \bar{\jmath} \bar{k}}}{J}+\left(b_{2}-b_{5}\right)^{A} \underline{R}_{\bar{\jmath} \bar{k}}-\frac{1}{2}\left(\bar{b}_{2}+\bar{b}_{5}\right)^{A}\left(\varepsilon_{\bar{\jmath}}^{\bar{m} \bar{n}} R_{\perp \bar{k} \bar{m} \bar{n}}\right) \\
& =\left(b_{2}+b_{5}\right)^{A} R_{\perp \bar{\jmath} \perp \bar{k}}-\frac{1}{2}\left(\bar{b}_{2}-\bar{b}_{5}\right)^{A}\left(\varepsilon_{\bar{j}}^{\bar{m} \bar{n}} R_{\bar{m} \bar{n} \bar{k} \perp}\right), \\
{ }^{T} \Phi_{\perp \bar{\jmath} \bar{k}} & \equiv \frac{{ }^{T} \Pi_{\perp \bar{\jmath} \bar{k}}}{J}+\left(b_{1}-b_{4}\right)^{T} \underline{R}_{\bar{\jmath} \bar{k}}+\frac{1}{2}\left(\bar{b}_{1}+\bar{b}_{2}\right)^{T}\left(\varepsilon_{\bar{j}}^{\bar{m} \bar{n}} R_{\perp \bar{k} \bar{m} \bar{n}}\right) \\
& =\left(b_{1}+b_{4}\right)^{T} R_{\perp \bar{\jmath} \perp \bar{k}}-\frac{1}{2}\left(\bar{b}_{1}-\bar{b}_{2}\right)^{T}\left(\varepsilon_{\bar{\jmath}}{ }^{\bar{m} \bar{n}} R_{\bar{m} \bar{n} \perp \bar{k}}\right) .
\end{aligned}
$$


The irreducible parts of $\Phi_{\bar{\imath} \bar{\jmath} \bar{k}}=-\Phi_{\bar{\jmath} \bar{k}}$ are the pseudoscalar, the vector and the traceless symmetric part, see (C.5):

$$
\begin{aligned}
& { }^{P} \Phi \equiv \frac{{ }^{P} \Pi}{J}+12 \bar{a}_{0}+\left(b_{2}-b_{3}\right) \varepsilon^{\bar{k} \bar{m} \bar{n}} R_{\perp \bar{k} \bar{m} \bar{n}}-\left(\bar{b}_{1}+2 \bar{b}_{2}+\bar{b}_{3}\right) \underline{R} \\
& =-\left(b_{2}+b_{3}\right) \varepsilon^{\bar{k} \bar{m} \bar{n}} R_{\bar{k} \bar{m} \bar{n} \perp}-2\left(\bar{b}_{2}-\bar{b}_{3}\right) R_{\perp \perp}, \\
& { }^{V} \Phi_{\bar{\imath}} \equiv \frac{V \Pi_{\bar{\imath}}}{J}-\left(b_{4}-b_{5}\right) R_{\perp \bar{\imath}}+\frac{1}{2}\left(\bar{b}_{2}+\bar{b}_{5}\right) \varepsilon^{\bar{k} \bar{m} \bar{n}} R_{\bar{\imath} \bar{k} \bar{m} \bar{n}} \\
& =\left(b_{4}+b_{5}\right) R_{\bar{\imath} \perp}-\left(\bar{b}_{2}-\bar{b}_{5}\right) \varepsilon_{\bar{\imath}}{ }^{\bar{k}} \bar{n} R_{\perp \bar{k} \perp \bar{n}}, \\
& { }^{T} \Phi_{\bar{\imath} \bar{\jmath} \bar{k}} \equiv \frac{{ }^{T} \Pi_{\bar{\imath} \bar{\jmath} \bar{k}}}{J}+\left(b_{1}-b_{2}\right)^{T} R_{\perp \bar{\jmath} \bar{k} \bar{\imath}}-{ }^{T} \mathcal{H}_{\bar{\imath} \bar{\jmath} \perp \bar{k}}^{\prime-}(\bar{R}) \\
& =\left(b_{1}+b_{2}\right)^{T} R_{\bar{\imath} \bar{\jmath} \perp \bar{k}}-\left(\bar{b}_{1}-\bar{b}_{2}\right)^{T}\left(\varepsilon_{\bar{\imath} \bar{\jmath}} \bar{n}{ }^{\Sigma} R_{\perp \bar{n} \perp \bar{k}}\right) .
\end{aligned}
$$

In Eqs. (3.12) and (3.13), the underlined objects do not contain velocities, $\underline{R}:=R^{\bar{m} \bar{n}} \bar{m} \bar{n}$

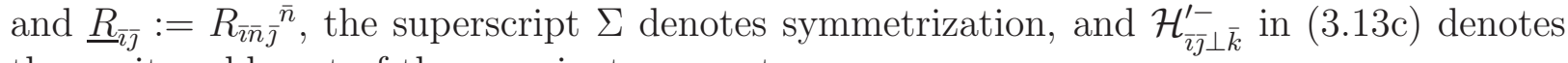
the parity odd part of the covariant momentum,

$$
{ }^{T} \mathcal{H}_{\bar{\imath} \jmath \perp \bar{k}}^{\prime-}(\bar{R})=-{ }^{T}\left\{\frac{1}{4} \varepsilon_{\bar{k}}^{\bar{m} \bar{n}}\left[\left(\bar{b}_{1}+2 \bar{b}_{2}+\bar{b}_{5}\right) R_{\bar{\imath} \bar{\jmath} \bar{m} \bar{n}}+\left(\bar{b}_{1}-\bar{b}_{5}\right) R_{\bar{m} \bar{n} \bar{\jmath}}\right]\right\} .
$$

Looking at the type of velocities appearing in the above equations, one can see that the critical parameters can be found by grouping these equations into suitably chosen pairs.

\section{Spin-0 pair}

Consider first Eqs. (3.12a) and (3.13a), which contain the same set of velocities, $R_{\perp \perp}$ and $\varepsilon^{\bar{k} \bar{m} \bar{n}} R_{\bar{k} \bar{m} \bar{n} \perp}$. They can be written in the matrix form as

$$
\left(\begin{array}{c}
{ }^{S} \Phi \\
{ }^{P} \Phi
\end{array}\right)=B_{0}\left(\begin{array}{c}
R_{\perp \perp} \\
\varepsilon^{\bar{k} \bar{m} \bar{n}} R_{\bar{k} \bar{m} \bar{n} \perp}
\end{array}\right)
$$

where

$$
\begin{aligned}
B_{0} & :=\left(\begin{array}{cc}
b_{4}+b_{6} & -\frac{1}{2}\left(\bar{b}_{2}-\bar{b}_{3}\right) \\
-2\left(\bar{b}_{2}-\bar{b}_{3}\right) & -\left(b_{2}+b_{3}\right)
\end{array}\right), \\
\operatorname{det} B_{0} & =-\left(b_{4}+b_{6}\right)\left(b_{2}+b_{3}\right)-\left(\bar{b}_{2}-\bar{b}_{3}\right)^{2} .
\end{aligned}
$$

In analogy to what we found in the previous subsection, the critical parameters are the eigenvalues of $B_{0}, c_{ \pm}\left(B_{0}\right)$, and the related column of the if-constraints reads

$$
{ }^{0} \Phi:=P_{0}^{-1}\left(\begin{array}{c}
S_{\Phi} \\
{ }^{P} \Phi
\end{array}\right)=D_{0} P_{0}^{-1}\left(\begin{array}{c}
R_{\perp \perp} \\
\varepsilon^{\bar{k} \bar{m} \bar{n}} R_{\bar{k} \bar{m} \bar{n} \perp}
\end{array}\right)
$$

were $P_{0}$ is the matrix that diagonalizes $B_{0}, D_{0}=P_{0}^{-1} B_{0} P_{0}$. 


\section{Spin-1 pair}

Similarly, after transforming ${ }^{A} \Phi_{\perp \bar{\jmath} \bar{j}}$ into ${ }^{A} \Phi_{\bar{k}}:=\varepsilon_{\bar{k}}{ }^{\bar{m}} \bar{n} \Phi_{\perp \bar{m} \bar{n}}$, Eq. (3.12b) becomes

$$
{ }^{A} \Phi_{\bar{\imath}}=\left(b_{2}+b_{5}\right) \varepsilon_{\bar{\imath}}{ }^{\bar{m} \bar{n}} R_{\perp \bar{m} \perp \bar{n}}+\left(\bar{b}_{2}-\bar{b}_{5}\right) R_{\bar{\imath} \perp},
$$

and the matrix form of Eqs. (3.16) and (3.13b) reads

$$
\left(\begin{array}{c}
{ }^{A} \Phi_{\bar{\imath}} \\
{ }^{V} \Phi_{\bar{\imath}}
\end{array}\right)=B_{1}\left(\begin{array}{c}
\varepsilon_{\bar{\imath}}{ }^{\bar{m}} \bar{n} R_{\perp \bar{m} \perp \bar{n}} \\
R_{\bar{\imath} \perp}
\end{array}\right)
$$

where

$$
\begin{aligned}
B_{1} & :=\left(\begin{array}{cc}
b_{2}+b_{5} & \bar{b}_{2}-\bar{b}_{5} \\
-\left(\bar{b}_{2}-\bar{b}_{5}\right) & b_{4}+b_{5}
\end{array}\right), \\
\operatorname{det} B_{1} & =\left(b_{4}+b_{5}\right)\left(b_{2}+b_{5}\right)+\left(\bar{b}_{2}-\bar{b}_{5}\right)^{2} .
\end{aligned}
$$

As before, the critical parameters are $c_{ \pm}\left(B_{1}\right)$, and the if-constraints are determined by the matrix $P_{1}$ that diagonalizes $B_{1}$,

$$
{ }^{1} \Phi_{\bar{\imath}}:=P_{1}^{-1}\left(\begin{array}{c}
{ }^{A} \Phi_{\bar{\imath}} \\
{ }^{V} \Phi_{\bar{\imath}}
\end{array}\right)=D_{1} P_{1}^{-1}\left(\begin{array}{c}
\varepsilon_{\bar{\imath}}^{\bar{m} \bar{n}} R_{\perp \bar{m} \perp \bar{n}} \\
R_{\bar{\imath} \perp}
\end{array}\right) .
$$

\section{Spin-2 pair}

To find the critical parameters in the spin-2 sector, it is convenient to replace ${ }^{T} \Phi_{\bar{\jmath} \bar{\jmath} \bar{k}}$ by the expression ${ }^{T} \Phi_{\bar{\imath} \bar{k}}:={ }^{T}\left(\varepsilon_{\bar{\imath}}{ }^{\bar{m}} \bar{n} \Phi_{\bar{m} \bar{n} \bar{k}}\right)$. Indeed, ${ }^{T} \Phi_{\bar{\imath} \bar{k}}$ refers to the same set of velocities that appears in Eq. (3.12c),

$$
{ }^{T} \Phi_{\bar{\imath} \bar{k}}=\left(b_{1}+b_{2}\right)^{T}\left(\varepsilon_{\bar{\imath}}^{\bar{m} \bar{n}} R_{\bar{m} \bar{n} \perp \bar{k}}\right)+2\left(\bar{b}_{1}-\bar{b}_{2}\right)^{T} R_{\perp \bar{\imath} \perp \bar{k}},
$$

which allows Eqs. (3.12c) and (3.19) to be written in the matrix form

$$
\left(\begin{array}{c}
{ }^{T} \Phi_{\perp \bar{\jmath}} \\
{ }^{T} \Phi_{\bar{\jmath} \bar{k}}
\end{array}\right)=B_{2}\left(\begin{array}{c}
{ }^{T} R_{\perp \bar{\jmath} \perp \bar{k}} \\
{ }^{T}\left(\varepsilon_{\bar{\jmath}}{ }^{\bar{m} \bar{n}} R_{\bar{m} \bar{n} \perp \bar{k}}\right.
\end{array}\right),
$$

where

$$
\begin{aligned}
B_{2} & :=\left(\begin{array}{cc}
b_{1}+b_{4} & -\frac{1}{2}\left(\bar{b}_{1}-\bar{b}_{2}\right) \\
2\left(\bar{b}_{1}-\bar{b}_{2}\right) & b_{1}+b_{2}
\end{array}\right), \\
\operatorname{det} B_{2} & =\left(b_{1}+b_{2}\right)\left(b_{1}+b_{4}\right)+\left(\bar{b}_{1}-\bar{b}_{2}\right)^{2} .
\end{aligned}
$$

Hence, the critical parameters are $c_{ \pm}\left(B_{2}\right)$, and the column of if-constraints has the form

$$
{ }^{2} \Phi_{\bar{\jmath} \bar{k}}:=P_{2}^{-1}\left(\begin{array}{c}
{ }^{T} \Phi_{\perp \bar{\jmath} \bar{k}} \\
{ }^{T} \Phi_{\bar{\jmath} \bar{k}}
\end{array}\right)=D_{2} P_{2}^{-1}\left(\begin{array}{c}
{ }^{T} R_{\perp \bar{\jmath} \perp \bar{k}} \\
{ }^{T}\left(\varepsilon_{\bar{\jmath}}^{\bar{m} \bar{n}} R_{\bar{m} \bar{n} \perp \bar{k}}\right)
\end{array}\right) .
$$

- The critical parameters in the curvature sector are $c_{ \pm}\left(B_{0}\right), c_{ \pm}\left(B_{1}\right)$, and $c_{ \pm}\left(B_{2}\right)$. 


\subsection{Critical parameters and if-constraints}

Since the if-constraints belong to irreducible representations of 3d rotations, they are characterized by a specific spin content. Their structure is best understood by grouping them into pairs with definite spin, as shown in Table 1. In this classification, the parity eigenvalues are absent since parity is not conserved.

Table 1. Critical parameters and if-constraints

\begin{tabular}{cll}
\hline \hline Spin & Critical parameters & If-constraints \\
\hline 0 & $a_{2}, c_{ \pm}\left(B_{0}\right)$ & ${ }^{S} \phi,\left({ }^{0} \Phi\right)_{ \pm}$ \\
1 & $c_{ \pm}(A), c_{ \pm}\left(B_{1}\right)$ & $\left(\phi_{\bar{k}}\right)_{ \pm},\left({ }^{1} \Phi_{\bar{k}}\right)_{ \pm}$ \\
2 & $a_{1}, c_{ \pm}\left(B_{2}\right)$ & ${ }^{T} \phi_{\bar{\imath} \bar{k}},\left({ }^{2} \Phi_{\bar{\imath} \bar{k}}\right)_{ \pm}$ \\
\hline \hline
\end{tabular}

The generic set of the critical parameters $c_{ \pm}(F), F=A, B_{0}, B_{1}, B_{2}$, is defined provided the parity odd parameters in $F$ do not vanish, see Appendix $\mathrm{D}$. Hence, the limit of the final expressions $c_{ \pm}(F)$ when these parameters tend to zero is not well defined. However, since in that case $F$ is already diagonal, one can identify $c_{ \pm}$directly from $F$.

The total number of the primary if-constraints is $10 \times 3=30$, the same as the number of the parallel canonical momenta (3.3). The if-constraints and the associated critical parameters have a decisive influence on the structure of the canonical Hamiltonian.

\section{Hamiltonian}

The procedure for constructing the canonical (and total) Hamiltonian in $\mathrm{PG}^{+}$is well known [7, 14, 15, 18, but its extension to PG, although in principle straightforward, is technically rather complicated.

Starting with the standard definition of the canonical Hamiltonian density,

$$
\mathcal{H}_{c}=\pi_{i}{ }^{\alpha} \partial_{0} b^{i}{ }_{\alpha}+\frac{1}{2} \Pi_{i j}{ }^{\alpha} \partial_{0} \omega^{i j}{ }_{\alpha}-b \mathcal{L},
$$

one can rewrite it in the Dirac-ADM form:

$$
\mathcal{H}_{c}=N \mathcal{H}_{\perp}+N^{\alpha} \mathcal{H}_{\alpha}-\frac{1}{2} \omega^{i j}{ }_{0} \mathcal{H}_{i j}+\partial_{\alpha} D^{\alpha},
$$

where $N$ and $N^{\alpha}$ are the lapse and shift functions (see Appendix [C), and

$$
\begin{aligned}
\mathcal{H}_{i j} & =2 \pi_{[i}{ }^{\alpha} b_{j] \alpha}+\nabla_{\alpha} \Pi_{i j}{ }^{\alpha}, \\
\mathcal{H}_{\alpha} & =\pi_{i}{ }^{\beta} T^{i}{ }_{\alpha \beta}+\frac{1}{2} \pi_{i j}{ }^{\beta} R^{i j}{ }_{\alpha \beta}-b^{k}{ }_{\alpha} \nabla_{\beta} \pi_{k}{ }^{\beta}, \\
\mathcal{H}_{\perp} & =\pi_{i}{ }^{\bar{k}} T^{i}{ }_{\perp \bar{k}}+\frac{1}{2} \Pi_{i j}{ }^{\bar{k}} R^{i j}{ }_{\perp \bar{k}}-J \mathcal{L}-n^{k} \nabla_{\beta} \pi_{k}{ }^{\beta}, \\
D^{\alpha} & =b^{i}{ }_{0} \pi_{i}{ }^{\alpha}+\frac{1}{2} \omega^{i j}{ }_{0} \Pi_{i j}{ }^{\alpha} .
\end{aligned}
$$

Since $\mathcal{H}_{\perp}$ is the only term that depends on the form of the Lagrangian, explicit construction of the whole $\mathcal{H}_{c}$ reduces just to the construction of its dynamical piece $\mathcal{H}_{\perp}$. In this process, 
we focus our attention on the "most dynamical" case when all the critical parameters are nonvanishing (that is, when none of the if-constraints becomes a true constraint). Such an assumption is sufficient for our study of the particle spectrum of PG. Extension of the formalism to include vanishing critical parameters is outlined in Appendix D.

\subsection{Torsion sector}

Isolating the torsion contribution to $\mathcal{L}_{G}$, one finds the corresponding part of $\mathcal{H}_{\perp}$,

$$
\mathcal{H}_{\perp}^{T}=\frac{1}{2} \phi_{i \perp \bar{k}} T^{i \perp \bar{k}}-J \overline{\mathcal{L}}_{T^{2}}-n^{k} \nabla_{\beta} \pi_{k}^{\beta},
$$

where $\overline{\mathcal{L}}_{T^{2}}=\mathcal{L}_{T^{2}}(\bar{T})$ does not contain velocities. In order to express the velocities in terms of the phase-space variables, we decompose the first term into four irreducible parts:

$$
\phi_{i \perp \bar{k}} T^{i \perp \bar{k}}=\phi_{\perp \bar{k}} T^{\perp \perp \bar{k}}+\frac{1}{2}{ }^{A} \phi_{\bar{\imath}} \varepsilon^{\bar{\imath}}{ }_{\bar{m} \bar{n}} T^{\bar{m} \bar{n} \perp}+{ }^{T} \phi_{\bar{\imath} \bar{k}}{ }^{T} T^{\bar{\imath} \perp \bar{k}}+\frac{1}{3}{ }^{S} \phi T_{\bar{k} \perp}{ }^{\bar{k}} .
$$

If $a_{1}, a_{2} \neq 0$, the velocities from the last two terms can be directly eliminated using Eqs. (3.6a) and (3.6d),

$$
\frac{1}{3}{ }^{S} \phi T_{\bar{k} \perp}{ }^{\bar{k}}+{ }^{T} \phi_{\bar{\imath} \bar{k}}{ }^{T} T^{\bar{\imath} \perp \bar{k}}=\frac{1}{6 a_{2}}{ }^{S} \phi^{S} \phi+\frac{1}{2 a_{1}}{ }^{T} \phi_{\bar{\imath} \bar{k}}{ }^{T} \phi^{\bar{\imath} \bar{k}} .
$$

Continuing with the first two terms in (4.4b), we note that, for $\operatorname{det} A \neq 0$, one can use the relation $A^{-1} \times($ (3.8a $)$ to eliminate the velocities. Introducing the notation $\varphi_{\bar{k}}:=\left(\phi_{\perp \bar{k}},{ }^{A} \phi_{\bar{k}}\right)^{T}$, the result takes a compact matrix form,

$$
\begin{aligned}
& \left(\phi_{\perp \bar{k}}, \quad \frac{1}{2}{ }^{A} \phi_{\bar{k}}\right)\left(\begin{array}{c}
T^{\perp \perp \bar{k}} \\
\varepsilon_{\bar{m} \bar{n}}^{\bar{k}} T^{\bar{m} \bar{n} \perp}
\end{array}\right)=\frac{3}{2 \operatorname{det} A} \varphi_{\bar{k}}^{T} T \varphi^{\bar{k}}, \\
& T:=\left(\begin{array}{cc}
2 a_{1}+a_{2} & \bar{a}_{1}-\bar{a}_{2} \\
\bar{a}_{1}-\bar{a}_{2} & -\left(a_{1}+2 a_{3}\right) / 2
\end{array}\right), \quad \operatorname{det} T=\frac{1}{2} \operatorname{det} A .
\end{aligned}
$$

Hence, the resulting form of $\mathcal{H}_{\perp}^{T}$ reads

$$
\begin{aligned}
\mathcal{H}_{\perp}^{T} & =\frac{1}{2} J \phi_{T}^{2}-J \overline{\mathcal{L}}_{T^{2}}-n^{k} \nabla_{\beta} \pi_{k}{ }^{\beta}, \\
\phi_{T}^{2} & :=\frac{1}{6 a_{2}}{ }^{S} \phi^{S} \phi+\frac{1}{2 a_{1}}{ }^{T} \phi_{\bar{\imath} \bar{k}}{ }^{T} \phi^{\bar{i} \bar{k}}+\frac{3}{2 \operatorname{det} A} \varphi_{\bar{k}}^{T} T \varphi^{\bar{k}},
\end{aligned}
$$

\subsection{Curvature sector}

In a similar manner, one finds the curvature contribution to $\mathcal{H}_{\perp}$ :

$$
\mathcal{H}_{\perp}^{R}=\frac{1}{4} \Phi_{i j \bar{k}} R^{i j \perp \bar{k}}-J \overline{\mathcal{L}}_{R^{2}}-a_{0} R^{\bar{m} \bar{n}} \bar{m} \bar{n}+\bar{a}_{0} \varepsilon^{\bar{m} \bar{n} \bar{k}} R_{\perp \bar{m} \bar{n} \bar{k}},
$$

where $\overline{\mathcal{L}}_{R^{2}}=\mathcal{L}_{R^{2}}(\bar{R})$ does not contain velocities, and

$$
\begin{aligned}
\Phi_{i j \bar{k}} R^{i j \perp \bar{k}}= & \frac{2}{3}{ }^{S} \Phi R_{\perp \perp}-{ }^{A} \Phi_{\bar{k}} \varepsilon^{\bar{k} \bar{m} \bar{n}} R_{\perp \bar{m} \perp \bar{n}}+2{ }^{T} \Phi_{\perp \bar{\jmath} \bar{k}}{ }^{T} R^{\perp \bar{\jmath} \perp \bar{k}}, \\
& -\frac{1}{6}{ }^{P} \Phi \varepsilon_{\overline{\bar{\imath}} \bar{k} \bar{k}} R^{\bar{\imath} \bar{\jmath} \perp \bar{k}}+{ }^{V} \Phi^{\bar{\imath}} R_{\bar{\imath} \bar{k} \perp}{ }^{\bar{k}}-\frac{1}{2}{ }^{T} \Phi^{\bar{\imath} \bar{k} T}\left(\varepsilon_{\bar{\imath}}{ }^{\bar{m} \bar{n}} R_{\bar{m} \bar{n} \perp \bar{k}} .\right.
\end{aligned}
$$


Summing up the scalar and pseudoscalar term from the expression (4.7b) and using the relation $B_{0}^{-1} \times(3.14 \mathrm{a})$ to eliminate the velocities, one obtains

$$
\begin{aligned}
& \frac{2}{3}{ }^{S} \Phi R_{\perp \perp}+\frac{1}{6}{ }^{P} \Phi \varepsilon_{\bar{\imath} \bar{\jmath} \bar{k}} R^{\bar{\imath} \bar{\jmath} \bar{k} \perp}=J \frac{1}{6 \operatorname{det} B_{0}}{ }^{(0)} \Phi^{T} R_{0}{ }^{(0)} \Phi \\
& R_{0}=\left(\begin{array}{cc}
-4\left(b_{2}+b_{3}\right) & 2\left(\bar{b}_{2}-\bar{b}_{3}\right) \\
2\left(\bar{b}_{2}-\bar{b}_{3}\right) & b_{4}+b_{6}
\end{array}\right), \quad \operatorname{det} R_{0}=4 \operatorname{det} B_{0},
\end{aligned}
$$

where ${ }^{(0)} \Phi^{T}:=\left({ }^{S} \Phi,{ }^{P} \Phi\right)$.

Similarly, the sum of the axial vector and vector term, combined with $B_{1}^{-1} \times(3.17 \mathrm{a})$, yields

$$
\begin{aligned}
& -{ }^{A} \Phi_{\bar{k}} \varepsilon^{\bar{k} \bar{m} \bar{n}} R_{\perp \bar{m} \perp \bar{n}}+{ }^{V} \Phi^{\bar{\imath}} R_{\bar{\imath} \perp}=-J \frac{1}{\operatorname{det} B_{1}}{ }^{(1)} \Phi_{\bar{\imath}}^{T} R_{1}{ }^{(1)} \Phi^{\bar{\imath}}, \\
& R_{1}=\left(\begin{array}{cc}
b_{4}+b_{5} & -\left(\bar{b}_{2}-\bar{b}_{5}\right) \\
-\left(\bar{b}_{2}-\bar{b}_{5}\right) & -\left(b_{2}+b_{5}\right)
\end{array}\right), \quad \operatorname{det} R_{1}=-\operatorname{det} B_{1},
\end{aligned}
$$

where ${ }^{(1)} \Phi_{\bar{\imath}}^{T}=\left({ }^{A} \Phi_{\bar{\imath}},{ }^{V} \Phi_{\bar{\imath}}\right)$.

Finally, using $B_{2}^{-1} \times(3.20 \mathrm{a})$, the sum of the two tensor terms is given by

$$
\begin{aligned}
& 2^{T} \Phi_{\perp \bar{\jmath} \bar{k}}{ }^{T} R^{\perp \bar{\jmath} \perp \bar{k}}-\frac{1}{2}{ }^{T} \Phi^{\bar{\imath} \bar{k} T}\left(\varepsilon_{\bar{\imath}}{ }^{\bar{m} \bar{n}} R_{\bar{m} \bar{n} \perp \bar{k}}\right)=J \frac{1}{4 \operatorname{det} B_{2}}{ }^{(2)} \Phi_{\bar{\imath} \bar{k}}^{T} R_{2}{ }^{(2)} \Phi^{\bar{\imath} \bar{k}}, \\
& R_{2}=\left(\begin{array}{cc}
4\left(b_{1}+b_{2}\right) & 2\left(\bar{b}_{2}-\bar{b}_{1}\right) \\
2\left(\bar{b}_{2}-\bar{b}_{1}\right) & -\left(b_{1}+b_{4}\right)
\end{array}\right), \quad \operatorname{det} R_{2}=-4 \operatorname{det} B_{2},
\end{aligned}
$$

where ${ }^{(2)} \Phi_{\bar{\imath} \bar{k}}^{T}:=\left({ }^{T} \Phi_{\perp \bar{\imath} \bar{k}},{ }^{T} \Phi_{\bar{\imath} \bar{k}}\right)$.

Summing up the above three contributions, one obtains the expression for $\mathcal{H}_{\perp}^{R}$ as

$$
\begin{aligned}
& \mathcal{H}_{\perp}^{R}=\frac{1}{4} J \Phi_{R}^{2}-J \mathcal{L}_{R^{2}}(\bar{R})-a_{0} R_{\bar{m} \bar{m} \bar{n}}^{\bar{m} \bar{n}}+\bar{a}_{0} \varepsilon^{\bar{m} \bar{n} \bar{k}} R_{\perp \bar{m} \bar{n} \bar{k}}, \\
& \Phi_{R}^{2}:=\frac{1}{6 \operatorname{det} B_{0}}{ }^{(0)} \Phi^{T} R_{0}{ }^{(0)} \Phi-{\frac{1}{\operatorname{det} B_{1}}}^{(1)} \Phi_{\bar{\imath}}^{T} R_{1}{ }^{(1)} \Phi^{\bar{\imath}}+\frac{1}{4 \operatorname{det} B_{2}}{ }^{(2)} \Phi_{\bar{\imath} \bar{k}}^{T} R_{2}{ }^{(2)} \Phi^{\bar{\imath} \bar{k}} .
\end{aligned}
$$

The complete expression $\mathcal{H}_{\perp}=\mathcal{H}_{\perp}^{T}+\mathcal{H}_{\perp}^{R}$ will be used in Sec. 7 to formulate the conditions for the positivity of energy of the isolated spin modes.

\subsection{Consistency conditions}

The complete canonical Hamiltonian of $\mathrm{PG}$, with $\mathcal{H}_{\perp}=\mathcal{H}_{\perp}^{T}+\mathcal{H}_{\perp}^{R}$, is calculated by assuming that none of the critical parameters is vanishing. In the next step, one can construct the total Hamiltonian that generates the temporal evolution of dynamical variables. Since the only primary constraints are the sure constraints (3.2), the total Hamiltonian is given by

$$
\mathcal{H}_{T}=\mathcal{H}_{c}+u^{i} \pi_{i}^{0}+\frac{1}{2} u^{i j} \Pi_{i j}{ }^{0},
$$

where $u^{i}$ and $u^{i j}$ are canonical multipliers. 
By construction, the components $\mathcal{H}_{i j}, \mathcal{H}_{\alpha}$ and $\mathcal{H}_{\perp}$ of the canonical Hamiltonian do not depend on the unphysical variables $b_{0}^{i}$ and $\omega^{i j}{ }_{0}$. Hence, by demanding the primary constraints to be preserved during the time evolution, one finds the set of secondary constraints,

$$
\mathcal{H}_{\perp} \approx 0, \quad \mathcal{H}_{\alpha} \approx 0, \quad \mathcal{H}_{i j} \approx 0 .
$$

General arguments, based on the existence of local Poincaré invariance, show that these constraints are first class [13], see also [28]. Hence, the Dirac consistency algorithm is completed at the level of the secondary constraints (4.13).

The present PG model has $N_{1}=20$ first-class constraints, and $N_{2}=0$ second-class constraints. Since the number of the Lagrangian variables is $N=40$ (16 tetrad, plus 24 connection components), the number of the Lagrangian degrees of freedom is $N^{*}=$ $\left(2 N-2 N_{1}-N_{2}\right) / 2=20$. They are the same as those found in the weak field approximation of PG: 2 massless spin- 2 modes and 18 massive torsion modes (two spin- 0 , six spin- 1 , and ten spin-2 modes). However, we shall show that not all of these degrees of freedom are physically acceptable, in contrast to earlier expectations [25]. To do that, we will first calculate the mass eigenvalues $m_{ \pm}^{2}(J)$ for the torsion modes with spin $J=0,1,2$.

\section{$5 \quad$ Linearized field equations}

In this section, we start our analysis of the particle spectrum of PG by deriving the weak field approximation of the gravitational field equations (2.4) around the Minkowski background $M_{4}$; for consistency, we assume $\Lambda_{0}=0$. Such an approximation is based on the following weak field expansion of the basic dynamical variables,

$$
b^{i}{ }_{\mu}=\delta_{\mu}^{i}+\tilde{b}_{\mu}^{i}+O_{2}, \quad \omega^{i j}{ }_{\mu}=\tilde{\omega}^{i j}{ }_{\mu}+O_{2} .
$$

To simplify the notation, we omit writing the tilde sign and the symbol $O_{2}$, with an implicit understanding of their effects. Furthermore, we find it technically convenient to use the following abbreviations:

$$
\begin{array}{ll}
A_{n}=a_{n}-a_{1}, & B_{n}=b_{n}-b_{1}, \\
\bar{A}_{n}=\bar{a}_{n}-\bar{a}_{1}, & \bar{B}_{n}=\bar{b}_{n}-\bar{b}_{1},
\end{array}
$$

\subsection{First field equation}

In the first field equation (2.4a), the covariant momentum associated to torsion has the form

$$
\begin{aligned}
\mathcal{H}_{i m n}= & 2 a_{1} T_{i m n}+\frac{2}{3} A_{2}\left(\eta_{i m} \mathcal{V}_{n}-\eta_{i n} \mathcal{V}_{m}\right)+2 A_{3} \varepsilon_{i m n l} \mathcal{A}^{l}, \\
& -\bar{a}_{1} T_{i r s} \varepsilon^{r s}{ }_{m n}-\frac{2}{3} \bar{A}_{2} \varepsilon_{i m n s} \mathcal{V}^{s}+2 \bar{A}_{3}\left(\eta_{i m} \mathcal{A}_{n}-\eta_{i n} \mathcal{A}_{m}\right),
\end{aligned}
$$

where $\bar{a}_{2}=\bar{a}_{3}$ yields $\bar{A}_{2}=\bar{A}_{3}$. Then, after calculating the linearized form of $E_{i}{ }^{\nu}$,

$$
E_{i}{ }^{\nu}=2 a_{0} G^{\nu}{ }_{i}-\bar{a}_{0}\left(R_{m n k i} \varepsilon^{m n k \nu}+h_{i}{ }^{\nu} X\right)=2 a_{0} G^{\nu}{ }_{i}-2 \bar{a}_{0} X_{i}^{\nu},
$$


the linearized (1ST) takes the form

$$
\begin{aligned}
\mathcal{E}_{i n}= & \partial^{m} \mathcal{H}_{i m n}-2 a_{0} G_{n i}+2 \bar{a}_{0} X_{i n} \\
= & -2 a_{1} \partial^{m} T_{i n m}+\frac{2}{3} A_{2}\left(\partial_{i} \mathcal{V}_{n}-\eta_{i n} \partial \mathcal{V}\right)-2 A_{3} \varepsilon_{i n m k} \partial^{m} \mathcal{A}^{k} \\
& +\frac{2}{3} \bar{A}_{2} \varepsilon_{i n m k} \partial^{m} \mathcal{V}^{k}+2 \bar{A}_{2}\left(\partial_{i} \mathcal{A}_{n}-\eta_{i n} \partial \mathcal{A}\right)-2 a_{0} G_{n i}+2\left(\bar{a}_{0}-\bar{a}_{1}\right) X_{i n}=0,
\end{aligned}
$$

where we used (E.3), and $\partial \mathcal{V}:=\partial_{i} \mathcal{V}^{i}, \partial \mathcal{A}:=\partial_{i} \mathcal{A}^{i}$.

\subsection{Second field equation}

Using the formulas obtained in the weak field approximation,

$$
\begin{aligned}
\nabla_{\mu}{ }^{L} H_{i j}{ }^{\mu n}= & 2 a_{0}\left(T^{n}{ }_{i j}-\delta_{i}^{n} \mathcal{V}_{j}+\delta_{j}^{n} \mathcal{V}_{i}\right) \\
& -\bar{a}_{0} \varepsilon_{i j}{ }^{r s}\left(T^{n}{ }_{r s}-\delta_{r}^{n} \mathcal{V}_{s}+\delta_{s}^{n} \mathcal{V}_{r}\right) \\
2 \mathcal{H}_{[i j] n}= & -\frac{4}{3}\left(2 a_{1}+a_{2}\right) \eta_{n[i} \mathcal{V}_{j]}+2\left(a_{1}+2 a_{3}\right) \varepsilon_{i j n k} \mathcal{A}^{k} \\
& -\frac{4}{3} \bar{A}_{2} \varepsilon_{i j n k} \mathcal{V}^{k}-4 \bar{A}_{3} \eta_{n[i} \mathcal{V}_{n]}
\end{aligned}
$$

the linearized form of (2ND) reads

$$
\begin{aligned}
\mathcal{E}_{i j n}= & \partial^{m} \mathcal{H}_{i j m n}^{\prime}+2 a_{0}\left(T^{n}{ }_{i j}-\delta_{i}^{n} \mathcal{V}_{j}+\delta_{j}^{n} \mathcal{V}_{i}\right) \\
& -\bar{a}_{0} \varepsilon_{i j}{ }^{r s}\left(T^{n}{ }_{r s}-\delta_{r}^{n} \mathcal{V}_{s}+\delta_{s}^{n} \mathcal{V}_{r}\right)+2 \mathcal{H}_{[i j] n}=0
\end{aligned}
$$

Using the double duality relations for the curvature, see Appendix C in Ref. [29], the term $\partial^{m} \mathcal{H}_{i j m n}^{\prime}$ is found to have the form

$$
\begin{aligned}
\partial^{m} \mathcal{H}_{i j m n}^{\prime}= & \left(b_{2}+b_{1}\right) \partial^{m}\left(\eta_{i r} \Psi_{j s}-\eta_{j r} \Psi_{i s}\right) \varepsilon^{r s}{ }_{m n}+\frac{1}{6} B_{3} \varepsilon_{i j m n} \partial^{m} X \\
& +\left(b_{4}+b_{1}\right)\left[\left(\partial_{i} \Phi_{j n}-\eta_{i n} \partial^{m} \Phi_{j m}\right)-(i \leftrightarrow j)\right]+\frac{1}{6} B_{6}\left(\eta_{j n} \partial_{i}-\eta_{i n} \partial_{j}\right) R \\
& +B_{5}\left[\left(\partial_{i} \hat{R}_{[j n]}-\eta_{i n} \partial^{m} \hat{R}_{[j m]}\right)-(i \leftrightarrow j)\right]-\bar{B}_{5} \partial^{m}\left(\eta_{i r} \hat{R}_{[j s]}-\eta_{j r} \hat{R}_{[i s]}\right) \varepsilon^{r s}{ }_{m n} \\
& +\left(\bar{b}_{2}-\bar{b}_{1}\right)\left[\left(\partial_{i} \Psi_{j n}-\eta_{i n} \partial^{m} \Psi_{j m}\right)-(i \leftrightarrow j)\right]+\frac{1}{6} \bar{B}_{3}\left(\eta_{j n} \partial_{i}-\eta_{i n} \partial_{j}\right) X \\
& -\left(\bar{b}_{4}-\bar{b}_{1}\right) \partial^{m}\left(\eta_{i r} \Phi_{j s}-\eta_{j r} \Phi_{i s}\right) \varepsilon^{r s}{ }_{m n}-\frac{1}{6} \bar{B}_{6} \varepsilon_{i j m n} \partial^{m} R .
\end{aligned}
$$

\section{Particle spectrum}

The particle spectrum of PG contains important information of its physical content. Recently, Karananas [25] made a detailed analysis of this problem by extending the spinprojection operator formalism, used earlier in the context of $\mathrm{PG}^{+}[12$, and applying it to

study the PG field excitations around the Minkowski background. His work resulted in the mass formulas for the spin-0, spin-1 and spin-2 massive torsion modes, together with the 
related restrictions on the parameter space, stemming from the requirements for the absence of ghosts and tachyons.

In this section, we study the same problem by analyzing the linearized field equations along the lines presented in [5]. The obtained results are tested by verifying their compatibility with the expressions for the critical parameters, found in the canonical analysis, whereas the absence of ghosts and tachyons is studied in the next section.

\subsection{Spin-0 modes}

The spin-0 sector is determined by the traces of the field equations $\mathcal{E}_{i n}, \partial^{i} \mathcal{E}_{i j n}$ and $\partial^{k}\left({ }^{\star} \mathcal{E}\right)_{k l n}$, where ${ }^{\star} \mathcal{E}_{k l n}:=(1 / 2) \varepsilon_{k l}{ }^{i j} \mathcal{E}_{i j n}$ is the dual of $\mathcal{E}_{i j n}$ :

$$
\begin{aligned}
& -a_{2} \partial V-3 \bar{a}_{2} \partial A+a_{0} R+\bar{a}_{0} X=0, \\
& \left(b_{4}+b_{6}\right) \square R+\left(\bar{b}_{3}-\bar{b}_{2}\right) \square X+4\left(2 a_{0}+a_{2}\right) \partial V+12\left(\bar{a}_{2}-\bar{a}_{0}\right) \partial A=0, \\
& \left(b_{2}+b_{3}\right) \square X-\left(\bar{b}_{3}-\bar{b}_{2}\right) \square R-12\left(a_{0}+2 a_{3}\right) \partial A+8\left(\bar{a}_{2}-\bar{a}_{0}\right) \partial V=0 .
\end{aligned}
$$

With $X=3 \partial A$, the first equation can be used to express $R$ in terms of $\partial \mathcal{V}$ and $\partial \mathcal{A}$, whereupon the remaining two equations are written in the matrix form as

$$
\begin{aligned}
& \left(K_{0} \square+4 a_{0} N_{0}\right) U=0, \\
& K_{0}=\left(\begin{array}{cc}
a_{2}\left(b_{4}+b_{6}\right) & -3 a_{0}\left(\bar{b}_{2}-\bar{b}_{3}\right)-3\left(\bar{a}_{0}-\bar{a}_{2}\right)\left(b_{4}+b_{6}\right) \\
a_{2}\left(\bar{b}_{2}-\bar{b}_{3}\right) & 3 a_{0}\left(b_{2}+b_{3}\right)-3\left(\bar{a}_{0}-\bar{a}_{2}\right)\left(\bar{b}_{2}-\bar{b}_{3}\right)
\end{array}\right), \\
& N_{0}=\left(\begin{array}{cc}
\left(2 a_{0}+a_{2}\right) & -3\left(\bar{a}_{0}-\bar{a}_{2}\right) \\
-2\left(\bar{a}_{0}-\bar{a}_{2}\right) & -3\left(a_{0}+2 a_{3}\right)
\end{array}\right), \quad U=\left(\begin{array}{c}
\partial \mathcal{V} \\
\partial \mathcal{A}
\end{array}\right) .
\end{aligned}
$$

The determinants of $K_{0}$ and $N_{0}$ are given by

$$
\begin{aligned}
& \operatorname{det} K_{0}=3 a_{0} a_{2}\left[\left(b_{4}+b_{6}\right)\left(b_{2}+b_{3}\right)+\left(\bar{b}_{2}-\bar{b}_{3}\right)^{2}\right], \\
& \operatorname{det} N_{0}=-3\left[\left(2 a_{0}+a_{2}\right)\left(a_{0}+2 a_{3}\right)+2\left(\bar{a}_{0}-\bar{a}_{2}\right)^{2}\right] .
\end{aligned}
$$

For $\operatorname{det} K_{0} \neq 0$, one can multiply (6.2a) by $K_{0}^{-1}$, and obtain the Klein-Gordon equation for the massive spin-0 torsion modes,

$$
\left(\square+M_{0}\right) U=0, \quad M_{0}=4 a_{0} K_{0}^{-1} N_{0} .
$$

The masses of these modes are given by the eigenvalues of the mass matrix $M_{0}$,

$$
\begin{aligned}
m_{ \pm}^{2}(0) & =\frac{1}{2}\left(\operatorname{tr} M_{0} \pm \sqrt{\left(\operatorname{tr} M_{0}\right)^{2}-4\left(\operatorname{det} M_{0}\right)}\right) \\
& =\frac{2 a_{0}}{\operatorname{det} K_{0}}\left(\operatorname{tr} f_{0}+\sqrt{\left(\operatorname{tr} f_{0}\right)^{2}-4 \operatorname{det} f_{0}}\right)
\end{aligned}
$$

where $f_{0}:=\left(\operatorname{det} K_{0}\right) K_{0}^{-1} N_{0}$, and

$$
\begin{aligned}
\operatorname{tr} f_{0}= & 3 a_{0}\left(2 a_{0}+a_{2}\right)\left(b_{2}+b_{3}\right)-12 a_{0}\left(\bar{a}_{0}-\bar{a}_{2}\right)\left(\bar{b}_{2}-\bar{b}_{3}\right) \\
& -3\left[a_{2}\left(a_{0}+2 a_{3}\right)+2\left(\bar{a}_{0}-\bar{a}_{2}\right)^{2}\right]\left(b_{4}+b_{6}\right), \\
\operatorname{det} f_{0}= & \left(\operatorname{det} K_{0}\right)\left(\operatorname{det} N_{0}\right) .
\end{aligned}
$$


It is interesting to note that $\operatorname{det} K_{0}$ is proportional to the product of two critical parameters, $a_{2}$ and $\operatorname{det} B_{0}$, characterizing the spin-0 sector of the set of if-constraints (see Table 1). Hence, when the critical parameters vanish, we have $\operatorname{det} K_{0}=0$, the mass eigenvalues (6.4) become infinite, and consequently, the spin-0 modes do not propagate. In the linear regime, this mechanism provides a Lagrangian description of the dynamical role of if-constraints.

As a further test of our mass formula (6.4), we calculated its form in the parity-even sector $\left(\bar{a}_{0}, \bar{a}_{n}, \bar{b}_{n}\right)=0$, and found the well-known result for the spin- $0^{ \pm}$torsion modes:

$$
m_{+}^{2}(0)=\frac{4 a_{0}\left(2 a_{0}+a_{2}\right)}{a_{2}\left(b_{4}+b_{6}\right)}, \quad m_{-}^{2}(0)=-\frac{4\left(a_{0}+2 a_{3}\right)}{\left(b_{2}+b_{3}\right)} .
$$

\subsection{Spin-1 modes}

To understand the linearized dynamics of the spin-1 sector, it is convenient to start with the antisymmetric part of $(1 \mathrm{ST}), \mathcal{E}_{[i j]}$, and its dual, ${ }^{\star} \mathcal{E}_{i j}$. Taking derivatives of these equations yields

$$
\begin{aligned}
& \frac{1}{3}\left(2 a_{1}+a_{2}\right)\left(\square \mathcal{V}_{j}-\partial_{j} \partial \mathcal{V}\right)+\bar{A}_{3}\left(\square \mathcal{A}_{j}-\partial_{j} \partial \mathcal{A}\right)+2 A_{0} \partial^{i} \hat{R}_{[i j]}+2 \bar{A}_{0} \partial^{i} X_{[i j]}=0 \\
& \left(a_{1}+2 a_{3}\right)\left(\square \mathcal{A}_{j}-\partial_{j} \partial \mathcal{A}\right)-\frac{2}{3} \bar{A}_{2}\left(\square \mathcal{V}_{j}-\partial_{j} \partial \mathcal{V}\right)-2 A_{0} \partial^{i} X_{[i j]}+2 \bar{A}_{0} \partial^{i} \hat{R}_{[i j]}=0 .
\end{aligned}
$$

Then, the solutions for $\partial^{m} \hat{R}_{[m i]}$ and $\partial^{m} X_{[m i]}$ are found to be given in the matrix form as

$$
\begin{gathered}
2\left(\begin{array}{cc}
-\partial^{m} \hat{R}_{[m i]} \\
\partial^{m} X_{[m i]}
\end{array}\right)=G\left(\square U_{i}-\partial_{i}(\partial U)\right), \quad U_{i}=\left(\begin{array}{c}
\mathcal{V}_{i} \\
\mathcal{A}_{i}
\end{array}\right), \quad g:=A_{0}^{2}+\bar{A}_{0}^{2}, \\
G:=\frac{1}{g}\left(\begin{array}{cc}
\frac{1}{3}\left[A_{0}\left(2 a_{1}+a_{2}\right)-2 \bar{A}_{0} \bar{A}_{2}\right] & {\left[A_{0} \bar{A}_{2}+\bar{A}_{0}\left(a_{1}+2 a_{3}\right)\right]} \\
-\frac{1}{3}\left[\bar{A}_{0}\left(2 a_{1}+a_{2}\right)+2 A_{0} \bar{A}_{2}\right] & -\left[\bar{A}_{0} \bar{A}_{2}-A_{0}\left(a_{1}+2 a_{3}\right)\right]
\end{array}\right) .
\end{gathered}
$$

Next, consider the trace of $(2 \mathrm{ND}), \eta^{j k} \mathcal{E}_{i j k}$, and of its dual, $\eta^{j k \star} \mathcal{E}_{i j k}$. Using the identities (E.4), these trace components take the form

$$
\begin{aligned}
& -2\left(b_{4}+b_{5}\right) \partial^{m} \hat{R}_{[m i]}+2\left(\bar{b}_{2}-\bar{b}_{5}\right) \partial^{m} X_{[m i]}+\frac{1}{2}\left(b_{4}+b_{6}\right) \partial_{i} R-\frac{1}{2}\left(\bar{b}_{2}-\bar{b}_{3}\right) \partial_{i} X \\
& \quad+2\left(2 a_{0}+a_{2}\right) \mathcal{V}_{i}-6\left(\bar{a}_{0}-\bar{a}_{2}\right) \mathcal{A}_{i}=0, \\
& -4\left(\bar{b}_{2}-\bar{b}_{5}\right) \partial^{m} \hat{R}_{[m i]}-4\left(b_{2}+b_{5}\right) \partial^{m} X_{[m i]}+\left(\bar{b}_{2}-\bar{b}_{3}\right) \partial_{i} R+\left(b_{2}+b_{3}\right) \partial_{i} X \\
& \quad-8\left(\bar{a}_{0}-\bar{a}_{2}\right) \mathcal{V}_{i}-12\left(a_{0}+2 a_{3}\right) \mathcal{A}_{i}=0 .
\end{aligned}
$$

Using the expressions for $\partial^{m} \hat{R}_{[m i]}$ and $\partial^{m} X_{[m i]}$ found in (6.5), and the expression for $R$ determined by the trace of (1ST), Eqs. (6.6) multiplied by $-2 g$ can be written in the matrix form as

$$
\left(K_{1} \square-4 g N_{1}\right) U_{i}+\left(L_{1}-K_{1}\right) \partial_{i}(\partial U)=0
$$


where

$$
\begin{aligned}
& K_{1}=B_{1}^{\prime}(g G), \quad B_{1}^{\prime}:=-2\left(\begin{array}{cc}
b_{4}+b_{5} & \bar{b}_{2}-\bar{b}_{5} \\
2\left(\bar{b}_{2}-\bar{b}_{5}\right) & -2\left(b_{2}+b_{5}\right)
\end{array}\right), \\
& N_{1}=\left(\begin{array}{cc}
\left(2 a_{0}+a_{2}\right) & -3\left(\bar{a}_{0}-\bar{a}_{2}\right) \\
-4\left(\bar{a}_{0}-\bar{a}_{2}\right) & -6\left(a_{0}+2 a_{3}\right)
\end{array}\right), \quad U_{i}=\left(\begin{array}{c}
\mathcal{V}_{i} \\
\mathcal{A}_{i}
\end{array}\right) \text {, } \\
& L_{1}=-\frac{g}{a_{0}}\left(\begin{array}{ll}
1 & 0 \\
0 & 2
\end{array}\right) K_{0}=-4 g N_{1} M_{0}^{-1} \text {. }
\end{aligned}
$$

The determinants of $K_{1}$ and $N_{1}$ are given by

$$
\begin{aligned}
& \operatorname{det} K_{1}=\frac{8}{3} g(\operatorname{det} A)\left(\operatorname{det} B_{1}\right), \\
& \operatorname{det} N_{1}=-6\left[\left(a_{0}+2 a_{3}\right)\left(2 a_{0}+a_{2}\right)+2\left(\bar{a}_{0}-\bar{a}_{2}\right)^{2}\right] .
\end{aligned}
$$

When $\operatorname{det} K_{1} \neq 0$, one can multiply Eq. (6.7a) by $K_{1}^{-1}$ and obtain the matrix Klein-Gordon equation for the massive spin-1 torsion modes,

$$
\begin{aligned}
& \left(\square+M_{1}\right) \tilde{U}_{i}=0, \quad M_{1}:=-4 g K_{1}^{-1} N_{1}, \\
& \tilde{U}_{i}:=U_{i}+M_{0}^{-1} \partial_{i} U, \quad \partial^{i} \tilde{U}_{i}=0 .
\end{aligned}
$$

The eigenvalues of the mass matrix $M_{1}$ are given by

$$
m_{ \pm}^{2}(1)=\frac{-2 g}{\operatorname{det} K_{1}}\left(\operatorname{tr} f_{1} \pm \sqrt{\left(\operatorname{tr} f_{1}\right)^{2}-4 \operatorname{det} f_{1}}\right)
$$

where $f_{1}:=\left(\operatorname{det} K_{1}\right) K_{1}^{-1} N_{1}$, and

$$
\begin{aligned}
& \operatorname{det} f_{1}=\left(\operatorname{det} N_{1}\right)\left(\operatorname{det} K_{1}\right) \\
& \operatorname{tr} f_{1}=4\left(b_{2}+b_{5}\right)\left[\left(2 a_{0}+a_{2}\right)\left[\left(a_{0}-a_{1}\right)\left(a_{1}+2 a_{3}\right)-\left(\bar{a}_{0}-\bar{a}_{1}\right)^{2}\right]+2\left(a_{0}-a_{1}\right)\left(\bar{a}_{0}-\bar{a}_{2}\right)^{2}\right] \\
& \quad+4\left(b_{4}+b_{5}\right)\left[\left(a_{0}+2 a_{3}\right)\left[\left(a_{0}-a_{1}\right)\left(2 a_{1}+a_{2}\right)-2\left(\bar{a}_{0}-\bar{a}_{1}\right)^{2}\right]+2\left(a_{0}-a_{1}\right)\left(\bar{a}_{0}-\bar{a}_{2}\right)^{2}\right] \\
& \quad+8\left(\bar{b}_{2}-\bar{b}_{5}\right)\left[-\left(2 a_{0}+a_{2}\right)\left(a_{0}+2 a_{3}\right)\left(\bar{a}_{0}-\bar{a}_{1}\right)+2\left[\left(a_{0}-a_{1}\right)^{2}+\left(\bar{a}_{0}-\bar{a}_{1}\right)^{2}\right]\left(\bar{a}_{0}-\bar{a}_{2}\right)\right. \\
& \left.\quad-2\left(\bar{a}_{0}-\bar{a}_{1}\right)\left(\bar{a}_{0}-\bar{a}_{2}\right)^{2}\right] .
\end{aligned}
$$

The determinant of $K_{1}$ is the product of two critical parameters associated to the spin-1 sector (see Table 1). A discussion of what happens when at least one of these parameters vanishes is given in Appendix D,

In the parity even sector, our mass formula (6.9) yields the familiar result for the spin- $1^{ \pm}$ torsion modes:

$$
m_{+}^{2}(1)=\frac{6\left(a_{0}-a_{1}\right)\left(a_{0}+2 a_{3}\right)}{\left(a_{1}+2 a_{3}\right)\left(b_{2}+b_{5}\right)}, \quad m_{-}^{2}(1)=\frac{6\left(a_{0}-a_{1}\right)\left(2 a_{0}+a_{2}\right)}{\left(2 a_{1}+a_{2}\right)\left(b_{4}+b_{5}\right)} .
$$




\subsection{Spin-2 modes}

Although, in principle, the analysis of the spin-2 sector is not much more complicated than the one for the spin-1 case, the fact that there are lots of variables makes the general procedure rather complex and difficult to follow. In Ref. [27], the mass eigenvalues of the spin-2 torsion modes were found by studying a class of exact wave solutions, defined by an ansatz that creates only the tensorial irreducible part of the torsion, whereas the vector and axial vector parts vanish. This motivates us to simplify the present discussion by considering a dynamical system with vanishing spin-0 and spin-1 modes, $\mathcal{V}_{i}=0$ and $\mathcal{A}_{i}=0$. The physical content of such a system is described solely by the spin- 2 tensor $t_{i j k}$ (Appendix A). Such a technical simplification does not influence the final result for the spin-2 mass eigenvalues.

The adopted assumptions have two additional consequences: $X=0$, which follows from $X=3 \partial \mathcal{A}$, and $R=0$, which follows from the trace of (1ST). To analyse the spin- 2 sector, we need the symmetrized version of (1ST),

$$
-a_{1} \Theta_{i k}-a_{0} \Phi_{i k}+\bar{A}_{0} \Psi_{i k}=0
$$

where $\Theta_{i k}:=\partial^{m} t_{i k m}=\partial^{m} T_{(i k) m}$, as follows from the definition (A.1) of $t_{i k m}$. Moreover, we also need two equations that follow from (2ND), $\partial^{m} \mathcal{E}_{m(i k)}$, and $\partial^{m}\left({ }^{\star} \mathcal{E}\right)_{m(i k)}$ :

$$
\begin{aligned}
& \left(b_{1}+b_{4}\right)\left[\square \Phi_{i k}-2 \partial_{(i} \partial^{m} \Phi_{k) m}\right]+\bar{B}_{2}\left[\square \Psi_{i k}-2 \partial_{(i} \partial^{m} \Psi_{k) m}\right] \\
& \quad-2 A_{0} \Theta_{i k}-2 \bar{A}_{0} \Psi_{i k}=0, \\
& \left(b_{1}+b_{2}\right)\left[\square \Psi_{i k}-2 \partial_{(i} \partial^{m} \Psi_{k) m}\right]-\bar{B}_{4}\left[\square \Phi_{i k}-2 \partial_{(i} \partial^{m} \Phi_{k) m}\right] \\
& \quad-2 \bar{A}_{0} \Theta_{i k}+2 A_{0} \Psi_{i k}=0 .
\end{aligned}
$$

Since $\Phi_{i k}$ has a nontrivial Riemannian part associated to the massless graviton, a proper description of the torsion spin-2 modes is obtained by using (6.10) to eliminate $\Phi_{i k}$ from Eqs. 6.11):

$$
\begin{aligned}
& \left(b_{1}+b_{4}\right) \square\left(-a_{1} \Theta_{i k}+\bar{A}_{0} \Psi_{i k}\right)+a_{0} \bar{B}_{2} \square \Psi_{i k}-2 a_{0}\left(A_{0} \Theta_{i k}+\bar{A}_{0} \Psi_{i k}\right)=0, \\
& a_{0}\left(b_{1}+b_{2}\right) \square \Psi_{i k}-\bar{B}_{2} \square\left(-a_{1} \Theta_{i k}+\bar{A}_{0} \Psi_{i k}\right)-2 a_{0}\left(\bar{A}_{0} \Theta_{i k}-A_{0} \Psi_{i k}\right)=0 .
\end{aligned}
$$

These equations can be compactly represented in the matrix form as

$$
\left(K_{2} \square+2 a_{0} N_{2}\right) U_{i k}=0,
$$

where

$$
\begin{aligned}
& K_{2}:=\left(\begin{array}{cc}
a_{1}\left(b_{1}+b_{4}\right) & -\bar{A}_{0}\left(b_{1}+b_{4}\right)-a_{0}\left(\bar{b}_{2}-\bar{b}_{1}\right) \\
-a_{1}\left(\bar{b}_{2}-\bar{b}_{1}\right) & \bar{A}_{0}\left(\bar{b}_{2}-\bar{b}_{1}\right)-a_{0}\left(b_{2}+b_{1}\right)
\end{array}\right), \\
& N_{2}:=\left(\begin{array}{cc}
A_{0} & \bar{A}_{0} \\
\bar{A}_{0} & -A_{0}
\end{array}\right), \quad U_{i k}:=\left(\begin{array}{c}
\Theta_{i k} \\
\Psi_{i k}
\end{array}\right) .
\end{aligned}
$$

For $\operatorname{det} K_{2} \neq 0$, Eq. (6.13) is equivalent to

$$
\left(\square+M_{2}\right) U_{i k}=0, \quad M_{2}:=2 a_{0} K_{2}^{-1} N_{2},
$$


where $M_{2}$ is the mass matrix of the spin-2 torsion mode.

The matrices $K_{2}$ and $N_{2}$ are of the same form as those found in Ref. [27], Eq. (4.50), up to inessential differences in conventions. Hence, the mass eigenvalues are also the same. Expressed in terms of the matrix $f_{2}=\left(\operatorname{det} K_{2}\right) K_{2}^{-1} N_{2}$, they are given by

$$
m_{ \pm}^{2}(2)=\frac{a_{0}}{\operatorname{det} K_{2}}\left(\operatorname{tr} f_{2} \pm \sqrt{\left(\operatorname{tr} f_{2}\right)^{2}-4 \operatorname{det} f_{2}}\right),
$$

where

$$
\begin{aligned}
\operatorname{det} f_{2}= & \left(\operatorname{det} K_{2}\right)\left(\operatorname{det} N_{2}\right), \\
\operatorname{tr} f_{2}= & -a_{0}\left(a_{0}-a_{1}\right)\left(b_{1}+b_{2}\right)-2 a_{0}\left(\bar{a}_{0}-\bar{a}_{1}\right)\left(\bar{b}_{2}-\bar{b}_{1}\right) \\
& +\left[-a_{1}\left(a_{0}-a_{1}\right)+\left(\bar{a}_{0}-\bar{a}_{1}\right)^{2}\right]\left(b_{1}+b_{4}\right) .
\end{aligned}
$$

As expected, the determinant of $K_{2}$ is proportional to the product of the critical parameters given in the third line of Table 1 ,

$$
\operatorname{det} K_{2}=-a_{0} a_{1} \operatorname{det} B_{2}, \quad \operatorname{det} N_{2}=-\left(A_{0}^{2}+\bar{A}_{0}^{2}\right) .
$$

In the parity-even sector, the above formulas produce the well-known result:

$$
m_{+}^{2}(2)=\frac{2 a_{0}\left(a_{0}-a_{1}\right)}{a_{1}\left(b_{1}+b_{4}\right)}, \quad m_{-}^{2}(2)=\frac{2\left(a_{0}-a_{1}\right)}{b_{1}+b_{2}} .
$$

The above procedure can be extended to the case with nonvanishing spin- 0 and spin- 1 terms. After a straightforward but rather clumsy calculation, we found that the new terms do not influence the mass eigenvalues, they only modify the spin-2 state $U_{i k}$. A compact form of the result reads

$$
U_{i k} \rightarrow \tilde{U}_{i k}:=Z\left[\bar{U}_{i k}-G \partial_{(i} U_{k)}+\frac{1}{3} H\left(M_{0}^{-1} \partial_{i} \partial_{k} U+\eta_{i k} U\right)\right],
$$

where

$$
\begin{aligned}
& Z:=\frac{1}{a_{1}}\left(\begin{array}{cc}
-a_{0} & \bar{A}_{0} \\
0 & a_{1}
\end{array}\right), \quad \bar{U}_{i k}:=\left(\begin{array}{c}
\Phi_{i k} \\
\Psi_{i k}
\end{array}\right), \\
& H:=\frac{1}{a_{0}}\left(\begin{array}{cc}
a_{2} & 3\left(\bar{a}_{2}-\bar{a}_{0}\right) \\
0 & -2 a_{0}
\end{array}\right) .
\end{aligned}
$$

The role of $Z$ is to replace $\Phi_{i k}$ in $\bar{U}_{i k}$ by its form obtained from the symmetrized (1ST). The spin-2 nature of $\tilde{U}_{i k}$ is ensured by the properties $\partial^{i} \tilde{U}_{i k}=0, \eta^{i k} \tilde{U}_{i k}=0$. In fact, these properties are sufficient to completely determine $\tilde{U}_{i k}$.

\subsection{Comparison with Karananas' mass formulas}

Our mass formulas are found to be consistent with the expressions for the canonical critical parameters, displayed in Table 1. A more detailed test can be conducted by comparing them to the recent calculations of Karananas [25. The first step in this direction is to compare the Lagrangian (5) in Ref. [25] with our expression (B.1). Although the procedure 
is straightforward, a number of misprints found in [25] complicate the process. Nevertheless, we established the following correspondence between the related parameters:

$$
\begin{array}{llrl}
a_{0} & =\lambda, & \bar{a}_{0} & =\Lambda=0, \\
a_{1} & =\lambda+t_{1}, & a_{2} & =2\left(-\lambda+t_{3}\right), \\
a_{3} & =\left(-\lambda+t_{2}\right) / 2, & & \\
\bar{a}_{1} & =-2 t_{5}, & \bar{a}_{2} & =\bar{a}_{3}=-t_{4}, \\
b_{1} & =4\left(r_{1}-r_{3}\right), & b_{2} & =4 r_{3}, \\
b_{3} & =4\left(r_{2}-r_{3}\right), & b_{4} & =4\left(r_{1}-r_{3}+r_{4}\right), \\
b_{5} & =4\left(r_{3}+r_{5}\right), & b_{6} & =4\left(r_{1}-r_{3}+3 r_{4}\right), \\
\bar{b}_{1} & =r_{7}-3 r_{8}, & \bar{b}_{2} & =\bar{b}_{4}=r_{7}+r_{8}, \\
\bar{b}_{3} & =\bar{b}_{6}=-4 r_{6}+r_{7}+r_{8}, & \bar{b}_{5}=-3 r_{7}+r_{8} .
\end{array}
$$

The remaining part of the comparison is rather simple. By substituting the above expressions into Eqs. (6.4) and (6.9), one finds that the resulting mass eigenvalues for the spin-0 and spin-1 torsion modes exactly reproduce the respective result that Karananas gives in his Appendix A. Moreover, we also found that, up to minor differences, our mass formula (6.15) for the spin-2 modes is in agreement with his result (A.3.5); see also subsection IV.E in Ref. [27]. Although the difference is small, it might be responsible for more serious discrepancies in the physical properties of the spin-2 modes, found in the next section.

\section{Physical restrictions on the space of parameters}

In this section, we study the physical requirements of the absence of ghosts $(E>0)$, the absence of tachyons $\left(m^{2}>0\right)$, and the reality $\left(m^{2}\right.$ real $)$, in the spectrum of torsion modes. Our approach is based on the Hamiltonian analysis developed in Secs. 3 and 4, subject to the assumption that all the critical parameters are nonvanishing, or equivalently, that all the torsion modes are propagating. In what follows, we shall examine whether such an assumption is compatible with the adopted physical requirements.

Our general strategy is the following. The conditions of the positivity of energy can be read from the dynamical component $\mathcal{H}_{\perp}^{R}$ of the canonical Hamiltonian, see Eq. (4.11). By introducing the matrices

$$
F_{J}:=\frac{1}{\operatorname{det} B_{J}} R_{J}, \quad J=0,1,2,
$$

these conditions can be expressed by demanding that the eigenvalues of $F_{j}$ be positive. Using the general formula for the eigenvalues of a $2 \times 2$ matrix, see (3.9), one can express these conditions in a more practical form as

$$
E_{J}>0: \quad \operatorname{det} F_{J}>0, \quad \operatorname{tr} F_{j}>0 .
$$

The absence of tachyons is effectively described by the conditions of positivity of the eigenvalues $m_{ \pm}^{2}(J)$ of the mass matrices $M_{J}$ :

$$
m_{ \pm}^{2}(J)>0: \quad \operatorname{det} M_{J}>0, \quad \operatorname{tr} M_{J}>0 .
$$


Moreover, the presence of square roots in the mass eigenvalues requires to check their reality:

$$
m_{ \pm}^{2}(J) \text { real: } \quad\left(\operatorname{tr} M_{J}\right)^{2}-4 \operatorname{det} M_{J}>0 .
$$

By applying these general physical criteria to the specific spin- $J$ sectors, one obtains a set of restrictions on the original Lagrangian parameters. An important goal of our analysis is to clarify the issue of their mutual (in)consistency. We shall always use $a_{0}>0$, the condition that ensures the correct limit to GR.

\subsection{Spin-0 sector}

\section{Positivity of energy}

The energy of the spin- 0 modes is positive if the eigenvalues of the matrix $F_{0}=R_{0} / \operatorname{det} B_{0}$ are positive. Since $\operatorname{det} R_{0}=4 \operatorname{det} B_{0}$, the first condition $\operatorname{det} F_{0}>0 \operatorname{implies}$ that $\operatorname{det} B_{0}>0$, or equivalently,

$$
\begin{aligned}
& \left(b_{2}+b_{3}\right)\left(b_{4}+b_{6}\right)+\left(\bar{b}_{2}-\bar{b}_{3}\right)^{2}<0, \\
& \Rightarrow \quad\left(b_{2}+b_{3}\right)\left(b_{4}+b_{6}\right)<0 .
\end{aligned}
$$

Then, the second condition takes the form $\operatorname{tr} R_{0}>0$. In combination with (7.4b), it yields the relations

$$
b_{2}+b_{3}<0, \quad b_{4}+b_{6}>0
$$

which coincide with those in appearing in $\mathrm{PG}^{+}$. The independent conditions are the condition (7.4a) and, for instance, the first one in (7.5)

$$
\left(b_{2}+b_{3}\right)\left(b_{4}+b_{6}\right)+\left(\bar{b}_{2}-\bar{b}_{3}\right)^{2}<0, \quad b_{2}+b_{3}<0 .
$$

These two conditions coincide with the first two relations found in Eq. (48) of Ref. [25] (the third relation is redundant).

\section{Positivity of $m_{ \pm}^{2}(0)$}

The mass matrix $M_{0}$ of the spin-0 torsion modes has the form (6.3),

$$
M_{0} / 4 a_{0}=K_{0}^{-1} N_{0}=\frac{1}{\operatorname{det} K_{0}} f_{0}, \quad \operatorname{det} K_{0}=-3 a_{0} a_{2} \operatorname{det} B_{0} .
$$

The positivity of its eigenvalues is expressed by the conditions $\operatorname{det} M_{0}>0$ and $\operatorname{tr} M_{0}>0$ :

$$
\frac{\operatorname{det} N_{0}}{\operatorname{det} K_{0}}>0, \quad \frac{1}{\operatorname{det} K_{0}} \operatorname{tr} f_{0}>0
$$

Since $\operatorname{det} B_{0}>0$, they take the form

$$
\begin{aligned}
& a_{2} \operatorname{det} N_{0}<0, \\
& a_{2} \operatorname{tr} f_{0}<0 .
\end{aligned}
$$


As shown in Appendix $\mathrm{F}$, these general conditions can be transformed into an unexpectedly simple form, in which the parameters $\left(b_{n} \bar{b}_{n}\right)$ are completely absent:

$$
a_{2}\left[\left(2 a_{0}+a_{2}\right)\left(a_{0}+2 a_{3}\right)+2\left(\bar{a}_{0}-\bar{a}_{2}\right)^{2}\right]>0, \quad a_{2}\left(2 a_{0}+a_{2}\right)>0 .
$$

Returning to the parameters introduced in (6.18), this result takes the form

$$
\left(t_{3}-\lambda\right)\left(t_{2} t_{3}+t_{4}^{2}\right)>0, \quad\left(t_{3}-\lambda\right) t_{3}>0 .
$$

The first formula is equivalent to Karananas' result [25], but the second one is different.

\section{$7.2 \quad$ Spin-1 sector}

\section{Positivity of energy}

Starting with $F_{1}:=R_{1} / \operatorname{det} B_{1}$ and using $\operatorname{det} R_{1}=-\operatorname{det} B_{1}$, the first condition for the positivity of energy, $\operatorname{det} F_{1}>0$, reads

$$
\begin{aligned}
& \operatorname{det} B_{1} \equiv\left(b_{2}+b_{5}\right)\left(b_{4}+b_{5}\right)+\left(\bar{b}_{2}-\bar{b}_{5}\right)^{2}<0, \\
& \Rightarrow \quad\left(b_{2}+b_{5}\right)\left(b_{4}+b_{5}\right)<0 .
\end{aligned}
$$

The second condition, written as $\operatorname{tr} R_{1}<0$ and combined with (7.11b), yields

$$
b_{2}+b_{5}>0, \quad b_{4}+b_{5}<0
$$

which is the $\mathrm{PG}^{+}$result. As the two independent conditions, we choose

$$
\left(b_{2}+b_{5}\right)\left(b_{4}+b_{5}\right)+\left(\bar{b}_{2}-\bar{b}_{5}\right)^{2}<0, \quad b_{4}+b_{5}<0 .
$$

Again, there is a complete agreement with the first two relations in Eq. (49) of [25], whereas the third relation is redundant.

\section{Positivity of $m_{ \pm}^{2}(1)$}

To make the technical exposition more compact, we introduce the following notation:

$$
\begin{array}{lll}
\mu_{2}:=2 a_{0}+a_{2}, & \mu_{3}:=a_{0} / 2+a_{3}, & k_{2}:=2 a_{1}+a_{2}, \quad k_{3}:=a_{1} / 2+a_{3} . \\
\operatorname{det} A=-2\left[k_{2} k_{3}+\left(\bar{a}_{1}-\bar{a}_{2}\right)^{2}\right], & \operatorname{det} N_{1}=-12\left[\mu_{2} \mu_{3}+\left(\bar{a}_{0}-\bar{a}_{2}\right)^{2}\right] .
\end{array}
$$

The mass matrix of the spin-1 torsion modes was found in subsection 6.2 ,

$$
M_{1}=-4 g K_{1}^{-1} N_{1}=-\frac{4 g}{\operatorname{det} K_{1}} f_{1}, \quad \operatorname{det} K_{1}=\frac{8}{3} g(\operatorname{det} A)\left(\operatorname{det} B_{1}\right),
$$

with $g \equiv A_{0}^{2}+\bar{A}_{0}^{2}$. The positivity of the mass eigenvalues is expressed by the requirements

$$
\frac{\operatorname{det} N_{1}}{\operatorname{det} K_{1}}>0, \quad \frac{1}{\operatorname{det} K_{1}} \operatorname{tr} f_{1}<0
$$


Since $\operatorname{det} B_{1}<0$, these conditions are equivalent to

$$
\begin{aligned}
& (\operatorname{det} A)\left(\operatorname{det} N_{1}\right)<0, \\
& (\operatorname{det} A) \operatorname{tr} f_{1}>0 .
\end{aligned}
$$

The expression for $\operatorname{tr} f_{1}$ is given in subsection 6.2, see also Appendix $\mathbb{E}$

A simple inspection of (7.16a) shows that it can be realized by $\operatorname{det} A<0, \operatorname{det} N_{1}>0$, or vice versa, whereas, as shown in Appendix $\mathrm{F}$ (7.16b) can be replaced by a much simpler expression. The resulting conditions, equivalent to (7.16), are defined in (F.7):

$$
\begin{array}{ll}
\text { (i) } & k_{2} k_{3}+\left(\bar{a}_{1}-\bar{a}_{2}\right)^{2}<0, \quad \mu_{2} \mu_{3}+\left(\bar{a}_{0}-\bar{a}_{2}\right)^{2}>0, \\
& \mu_{3} k_{2} A_{0}-2 \mu_{3} \bar{A}_{0}^{2}+A_{0}\left(\bar{a}_{0}-\bar{a}_{2}\right)^{2}<0, \\
\text { (ii) } & k_{2} k_{3}+\left(\bar{a}_{1}-\bar{a}_{2}\right)^{2}>0, \quad \mu_{2} \mu_{3}+\left(\bar{a}_{0}-\bar{a}_{2}\right)^{2}<0, \\
& \mu_{3} k_{2} A_{0}-2 \mu_{3} \bar{A}_{0}^{2}+A_{0}\left(\bar{a}_{0}-\bar{a}_{2}\right)^{2}>0 .
\end{array}
$$

As before, they do not depend on the parameters $\left(b_{n}, \bar{b}_{n}\right)$. Going over to the parameters defined in (6.18), the relations (i) read

$$
\begin{aligned}
& \left(t_{1}+t_{2}\right)\left(t_{1}+t_{3}\right)+\left(t_{4}-2 t_{5}\right)^{2}<0, \quad t_{2} t_{3}+t_{4}^{2}>0, \\
& t_{2}\left(t_{1}^{2}+4 t_{5}^{2}\right)+t_{1}\left(t_{2} t_{3}+t_{4}^{2}\right)>0 .
\end{aligned}
$$

The first two inequalities in the set (i) coincide with those found in Ref. [25], the third one is a bit different, but the whole complementary set (ii) is missing.

\section{$7.3 \quad$ Spin-2 sector}

\section{Positivity of energy}

The first condition for the positivity of the eigenvalues of $F_{2}=R_{2} / \operatorname{det} B_{2}, \operatorname{det} F_{2}>0$, combined with $\operatorname{det} R_{2}=-4 \operatorname{det} B_{2}$, takes the form

$$
\begin{aligned}
& \operatorname{det} B_{2} \equiv\left(b_{1}+b_{2}\right)\left(b_{1}+b_{4}\right)+\left(\bar{b}_{2}-\bar{b}_{1}\right)^{2}<0, \\
& \Rightarrow \quad\left(b_{1}+b_{2}\right)\left(b_{1}+b_{4}\right)<0 .
\end{aligned}
$$

The second condition combined with (7.18b) yields relations that are also valid in $\mathrm{PG}^{+}$,

$$
b_{1}+b_{2}<0, \quad b_{1}+b_{4}>0 .
$$

The two independent conditions are

$$
\left(b_{1}+b_{2}\right)\left(b_{1}+b_{4}\right)+\left(\bar{b}_{2}-\bar{b}_{1}\right)^{2}<0, \quad b_{1}+b_{2}<0 .
$$

Comparing these conditions to the first two relations in Eq. (50) of Ref. [25], one finds a complete agreement (the third relation is redundant). 


\section{Positivity of $m_{ \pm}^{2}(2)$}

The mass matrix for the spin-2 modes is found in subsection 6.3.

$$
\begin{aligned}
& M_{2}=2 a_{0} K_{2}^{-1} N_{2}=\frac{2 a_{0}}{\operatorname{det} K_{2}} f_{2}, \quad \operatorname{det} K_{2}=-a_{0} a_{1} \operatorname{det} B_{2}, \\
& \operatorname{det} N_{2}=-\left[\left(a_{0}-a_{1}\right)^{2}+\left(\bar{a}_{0}-\bar{a}_{1}\right)^{2}\right] .
\end{aligned}
$$

The positivity of the mass eigenvalues is expressed by the requirements

$$
\frac{\operatorname{det} N_{2}}{\operatorname{det} K_{2}}>0, \quad \frac{2 a_{0}}{\operatorname{det} K_{2}} \operatorname{tr} f_{2}>0 .
$$

The condition $\operatorname{det} N_{2}<0$ implies $\operatorname{det} K_{2}<0$, whereupon, relying on $\operatorname{det} B_{2}<0$, one obtains

$$
\begin{aligned}
& a_{1}<0, \\
& \operatorname{tr} f_{2}<0,
\end{aligned}
$$

where $\operatorname{tr} f_{2}$ is calculated in subsection 6.3 .

\section{Is the spin-2 sector free of ghosts and tachyons?}

Let us recall that in $\mathrm{PG}^{+}$, the conditions $a_{1}<0$ and $b_{1}+b_{2}<0$ imply $\operatorname{tr} f_{2}>0$, so that one of the two spin- $2^{ \pm}$modes is always a tachyon, as is well known. In what follows, we will prove, somewhat unexpectedly, that the same conclusion also holds in the general PG.

To show this, we rewrite $\operatorname{tr} f_{2}$ in a compact notation as

$$
\operatorname{tr} f_{2}=\alpha_{2}\left(b_{1}+b_{2}\right)+\beta_{2}\left(\bar{b}_{2}-\bar{b}_{1}\right)+\gamma_{2}\left(b_{1}+b_{4}\right), \quad \alpha_{2}<0,
$$

where the coefficients $\alpha_{2}, \beta_{2}$ and $\gamma_{2}$ can be read from Eq. (6.15b),

$$
\begin{aligned}
& \alpha_{2}=-a_{0}\left(a_{0}-a_{1}\right), \quad \beta_{2}=-2 a_{0}\left(\bar{a}_{0}-\bar{a}_{1}\right)^{2}, \\
& \gamma_{2}=-a_{1}\left(a_{0}-a_{1}\right)+\left(\bar{a}_{0}-\bar{a}_{2}\right)^{2},
\end{aligned}
$$

and $\alpha_{2}<$ follows from (7.22a). Since $b_{1}+b_{4}>0$, one finds

$$
\frac{\operatorname{tr} f_{2}}{b_{1}+b_{4}}=\alpha_{2} \frac{b_{1}+b_{2}}{b_{1}+b_{4}}+\beta_{2} \frac{\bar{b}_{2}-\bar{b}_{1}}{b_{1}+b_{4}}+\gamma_{2} \text {. }
$$

Having in mind the first relation in (7.20), written as

$$
\frac{b_{1}+b_{2}}{b_{1}+b_{4}}+x^{2}<0, \quad x:=\frac{\bar{b}_{2}-\bar{b}_{1}}{b_{1}+b_{4}},
$$

we find it useful to rewrite (7.23b) in an equivalent form,

$$
\begin{aligned}
& \frac{\operatorname{tr} f_{2}}{b_{1}+b_{4}}=\alpha_{2}\left(\frac{b_{1}+b_{2}}{b_{1}+b_{4}}+x^{2}\right)+F_{2}(x), \\
& F_{2}(x):=-\alpha_{2} x^{2}+\beta_{2} x+\gamma_{2} .
\end{aligned}
$$


A critical argument in our analysis comes from the observation that the discriminant of the quadratic function $F_{2}(x), \Delta_{2}=\beta_{2}^{2}+4 \alpha_{2} \gamma_{2}$, is automatically negative,

$$
\Delta_{2}=4 a_{0} a_{1}\left[\left(\bar{a}_{0}-\bar{a}_{1}\right)^{2}+\left(a_{0}-a_{1}\right)^{2}\right]=-4 a_{0} a_{1} \operatorname{det} N_{2}<0 .
$$

Next, since $\alpha_{2}<0$ (the parabola $F_{2}$ opens upward) and $\Delta_{2} / \alpha_{2}>0$ (minimum of $F_{2}$ is positive), it follows that $F_{2}(x)>0$ for any $x$. Hence, using (17.20), one obtains the result

$$
\operatorname{tr} f_{2}>\left(b_{1}+b_{4}\right) F_{2}(x)>0,
$$

which contradicts to (7.22b). Thus

S3. The two no-tachyon conditions, (7.22a $)$ and (7.22b), are mutually exclusive; hence, the two spin-2 torsion modes cannot be simultaneously physical.

Such a conclusion is not in agreement with the result found by Karananas [25].

\section{No-ghost conditions: spin-2 versus spin-1 sector}

The no-ghost conditions for spin-1 and spin-2 sectors are in contradiction to each other. Indeed, (7.12) implies that $b_{2}>b_{4}$, whereas (7.19) implies that $b_{4}>b_{2}$. Hence, only one of these two sectors can be physical. The result is in agreement with the Corrigendum in [25].

\subsection{Reality conditions}

The structure of the general reality conditions (7.3) looks rather cumbersome. However, after replacing $\left|\operatorname{tr} f_{0}\right|,\left|\operatorname{tr} f_{1}\right|$, and $\left|\operatorname{tr} f_{2}\right|$ with their minimal values, calculated from the inequalities ( $(\underline{F .3}),(\underline{F .6})$, and (7.25), respectively, the reality conditions (17.3) transform into

$$
\begin{array}{ll}
\text { spin 0: } & \left(b_{4}+b_{6}\right)^{2} a_{2} \operatorname{det} N_{0}+12 a_{0}\left(2 a_{0}+a_{2}\right)^{2} \operatorname{det} B_{0}<0, \\
\text { spin 1: } & g\left(b_{2}+b_{5}\right)^{2}(\operatorname{det} A)\left(\operatorname{det} N_{1}\right)-24 \alpha_{1}^{2} \operatorname{det} B_{1}<0, \\
\text { spin 2: } & \left(b_{1}+b_{4}\right)^{2} a_{1} \operatorname{det} N_{2}+4 a_{0}\left(a_{0}-a_{1}\right)^{2} \operatorname{det} B_{2}>0,
\end{array}
$$

see Sec. 6 and Appendix E. These formulas are much simpler than (7.3), but they represent only sufficient conditions for the reality of the corresponding mass eigenvalues.

\section{Summary and conclusions}

In this paper, we investigated generic aspects of the Hamiltonian structure of the general parity-violating PG, and used them to study the torsion particle spectrum [30].

Making use of Dirac's Hamiltonian approach, we identified the set of all if-constraints, the expressions that become true constraints if the corresponding critical parameters $c_{n}$ vanish. Both the if-constraints and the associated critical parameters have a crucial influence on the PG dynamics. Then, we constructed the generic form of the canonical Hamiltonian $\mathcal{H}_{c}$, determined by taking all the critical parameters to be nonvanishing. An extension of the procedure to allow for a proper treatment of the vanishing critical parameters is outlined in Appendix D. 
Apart from being important by itself, the Hamiltonian structure introduced here turns out to be intrinsically related to the particle spectrum of PG. To examine that subject, we first calculated the mass eigenvalues $m_{ \pm}^{2}(J)$ of the torsion modes with spin $J=0,1$, and 2, relying on the weak field approximation of the gravitational field equations around $M_{4}$. As a test of the results, we verified that $m_{ \pm}^{2}(J)$ are proportional to the inverse critical parameters $1 / c_{n}$. As a consequence, whenever some of $c_{n}$ vanish, the corresponding values of $m_{ \pm}^{2}(J)$ become infinite, thereby preventing the associated torsion modes to propagate. This is consistent with the canonical effects of the vanishing critical parameters in $\mathrm{PG}^{+}$ (in the weak field approximation). A comparison of our mass formulas to those found by Karananas 25] leads to the following conclusions.

(k1) For the spin-0 and spin-1 torsion modes, our results confirm those of Karananas.

(k2) For the spin-2 modes, there are certain differences, noted already in Ref. [27.

The absence of ghosts (positivity of energy) in the particle spectrum is ensured by demanding the positivity of the specific spin- $J$ terms in the canonical Hamiltonian, whereas the conditions for the absence of tachyons are defined by the requirement $m_{ \pm}^{2}(J)>0$. A detailed analysis shows that these requirements can be formulated as follows:

$$
\begin{array}{ll}
\text { Spin 0: } & \left(b_{2}+b_{3}\right)\left(b_{4}+b_{6}\right)+\left(\bar{b}_{2}-\bar{b}_{3}\right)^{2}<0, \quad b_{2}+b_{3}<0, \\
& a_{2}\left[\left(2 a_{0}+a_{2}\right)\left(a_{0} / 2+a_{3}\right)+\left(\bar{a}_{0}-\bar{a}_{2}\right)^{2}\right]<0, \quad a_{2}\left(2 a_{0}+a_{2}\right)>0 .
\end{array}
$$

Spin 1: $\left(b_{2}+b_{5}\right)\left(b_{4}+b_{5}\right)+\left(\bar{b}_{2}-\bar{b}_{5}\right)^{2}<0, \quad b_{4}+b_{5}<0$,

(i) $\left(2 a_{1}+a_{2}\right)\left(a_{1} / 2+a_{3}\right)+\left(\bar{a}_{1}-\bar{a}_{2}\right)^{2}<0, \quad\left(2 a_{0}+a_{2}\right)\left(a_{0} / 2+a_{3}\right)+\left(\bar{a}_{0}-\bar{a}_{2}\right)^{2}>0$, $\left(a_{0}-a_{1}\right)\left[\left(a_{0} / 2+a_{3}\right)\left(2 a_{1}+a_{2}\right)+\left(\bar{a}_{0}-\bar{a}_{2}\right)^{2}\right]-2\left(a_{0} / 2+a_{3}\right)\left(\bar{a}_{0}-\bar{a}_{1}\right)^{2}<0 ;$

(ii) an alternative set of conditions, obtained by (i) $\rightarrow(-1) \times(\mathrm{i})$.

Spin 2: $\left(b_{1}+b_{2}\right)\left(b_{1}+b_{4}\right)+\left(\bar{b}_{2}-\bar{b}_{1}\right)^{2}<0, \quad b_{1}+b_{2}<0$, the conditions for the absence of tachyons are mutually exclusive.

The results for the absence of ghosts (first line in each spin sector) are identical to those of Karananas, whereas the formulas describing the absence of tachyons show a number of less or more serious differences. In particular, the whole set of conditions (ii) in the spin-1 sector is missing in Karananas' analysis, but the most important difference is found in the spin-2 sector, where the two conditions for the absence of tachyons are in contradiction to each other, in contrast to Karananas' conclusion.

The presence of square roots in the expressions for the mass eigenvalues $m_{ \pm}^{2}(J)$ requires to verify their reality. A sufficient form of of the reality conditions, compactly presented at the end of Sec. 7, is much simpler than their general form.

In conclusion, our analysis clarifies the structure of the particle spectrum of the general PG by improving the results found by Karananas, in particular the status of the spin-2 sector. On the other hand, elements of the Hamiltonian structure introduced here, including its extension to the case of vanishing critical parameters outlined in Appendix $\mathrm{D}$, are a good starting point for further investigation of the full nonlinear dynamics of PG. 


\section{Acknowledgments}

We are very grateful to Georgios Karananas for his readiness to discuss all aspects of his earlier work, and for a careful reading of the manuscript. We would also like to thank Friedrich Hehl for useful comments. This work was partially supported by the Serbian Science Foundation under Grant No. 171031.

\section{A Irreducible decomposition of the field strengths}

The torsion tensor has three irreducible pieces:

$$
\begin{aligned}
{ }^{(2)} T_{i m n} & =\frac{1}{3}\left(\eta_{i m} \mathcal{V}_{n}-\eta_{i n} \mathcal{V}_{m}\right) \\
{ }^{(3)} T_{i m n} & =\varepsilon_{i m n k} \mathcal{A}^{k} \\
{ }^{(1)} T_{i m n} & =T_{i m n}-{ }^{(2)} T_{i m n}-{ }^{(3)} T_{i m n}=\frac{4}{3} t_{i[m n]}
\end{aligned}
$$

where

$$
\begin{aligned}
& \mathcal{V}_{n}:=T_{k n}^{k}, \quad \mathcal{A}_{k}:=\frac{1}{6} \varepsilon_{k r s t} T^{r s t} \\
& t_{i m n}:=T_{(i m) n}+\frac{1}{3} \eta_{n(i} \mathcal{V}_{m)}-\frac{1}{3} \eta_{i m} \mathcal{V}_{n}
\end{aligned}
$$

The Riemann-Cartan curvature tensor can be decomposed into six irreducible pieces:

$$
\begin{aligned}
& { }^{(2)} R_{i j m n}=\frac{1}{2}\left(\eta_{i k} \Psi_{j l}-\eta_{j k} \Psi_{i l}\right) \varepsilon^{k l}{ }_{m n}, \\
& { }^{(3)} R_{i j m n}=\frac{1}{12} X \varepsilon_{i j m n}, \\
& { }^{(4)} R_{i j m n}=\frac{1}{2}\left(\eta_{i m} \Phi_{j n}-\eta_{j m} \Phi_{i n}\right)-(m \leftrightarrow n), \\
& { }^{(5)} R^{i j}=\frac{1}{2}\left(\eta_{i m} \hat{R}_{[j n]}-\eta_{j m} \hat{R}_{[i n]}\right)-(m \leftrightarrow n), \\
& { }^{(6)} R_{i j m n}=\frac{1}{12} R\left(\eta_{i m} \eta_{j n}-\eta_{j m} \eta_{i m}\right), \\
& { }^{(1)} R_{i j m n}=R_{i j m n}-\sum_{a=2}{ }^{(a)} R_{i j m n},
\end{aligned}
$$

where

$$
\begin{array}{rlrl}
\hat{R}_{i m} & :=\operatorname{Ric}_{i m}=R_{i n m}{ }^{n}, & R & :=\operatorname{Ric}^{m}{ }_{m}, \\
X_{i j} & :=\frac{1}{2} R_{i k m n} \varepsilon^{k m n}{ }_{j}, & X:=X_{n}^{n}, \\
\Phi_{i j} & :=\operatorname{Ric}_{(i j)}-\frac{1}{4} \eta_{i j} R, & & \Psi_{i j}:=X_{(i j)}-\frac{1}{4} \eta_{i j} X .
\end{array}
$$

The above definitions are the tensor counterparts of the corresponding formulas given in terms of the differential forms, see [27, 29]. They imply the following relations characterizing 
the parity-odd sector:

$$
\begin{aligned}
& T^{i j k \star(2)} T_{i j k}=T^{i j k \star(3)} T_{i j k}, \\
& R^{i j k l \star(2)} R_{i j k l}=R^{i j k l \star(4)} R_{i j k l}, \\
& R^{i j k l \star(3)} R_{i j k l}=R^{i j k l \star(6)} R_{i j k l},
\end{aligned}
$$

and also

$$
\begin{aligned}
& T^{i j k \star(1)} T_{i j k}={ }^{(1)} T^{i j k \star(1)} T_{i j k}, \\
& R^{i j k l \star(1)} R_{i j k l}={ }^{(1)} R^{i j k l \star(1)} R_{i j k l}, \\
& R^{i j k l \star(5)} R_{i j k l}={ }^{(5)} R^{i j k l \star(5)} R_{i j k l} .
\end{aligned}
$$

\section{B Alternative form of the Lagrangian}

In this appendix, we rewrite our Lagrangian (2.6) in an equivalent form that allows an easier comparison to the literature [22, 25]:

$$
\begin{aligned}
\mathcal{L}_{G}= & -\left(a_{0} R+2 \Lambda_{0}+\bar{a}_{0} X\right)+\mathcal{L}_{T^{2}}+\mathcal{L}_{R^{2}}, \\
\mathcal{L}_{T^{2}}= & h_{1} T^{i j k} T_{i j k}+h_{2} T^{i m n} T_{n m i}+h_{3} V_{m} V^{n} \\
& +\varepsilon^{m n k l}\left(\bar{h}_{4} T^{i}{ }_{m n} T_{i k l}+\bar{h}_{5} T_{m n}{ }^{i} T_{k l i}\right), \\
\mathcal{L}_{R^{2}}= & \frac{1}{2}\left(f_{1} R^{i j m n} R_{i j m n}+f_{2} R^{i j m n} R_{i m j n}+f_{3} R^{i j m n} R_{m n i j}\right. \\
& \left.+f_{4} \operatorname{Ric}^{i m} \operatorname{Ric}_{i m}+f_{5} \operatorname{Ric}^{i m} \operatorname{Ric}_{m i}+f_{6} R^{2}\right) \\
& +\frac{1}{2} \varepsilon^{m n k l}\left(\bar{f}_{7} R_{m n k l} R+\bar{f}_{8} R_{i j m n} R^{i j}{ }_{k l}\right. \\
& \left.+\bar{f}_{9} R_{m n i j} R_{k l}{ }^{i j}+\bar{f}_{10} R_{m n i j} R^{i j}{ }_{k l}\right) .
\end{aligned}
$$

The parameters $\left(h_{n}, \bar{h}_{n}\right)$ and $\left(f_{n}, \bar{f}_{n}\right)$ can be expressed in terms of the "irreducible" parameters appearing in (2.6) as follows:

$$
\begin{aligned}
& h_{1}=\frac{1}{6}\left(2 a_{1}+a_{3}\right), \quad h_{2}=\frac{1}{3}\left(a_{1}-a_{3}\right), \quad h_{3}=-\frac{1}{3}\left(a_{1}-a_{2}\right), \\
& \bar{h}_{4}:=-\frac{1}{24}\left(4 \bar{a}_{1}+\bar{a}_{2}+\bar{a}_{3}\right), \quad \bar{h}_{5}:=-\frac{1}{6}\left(2 \bar{a}_{1}-\bar{a}_{2}-\bar{a}_{3}\right),
\end{aligned}
$$

and

$$
\begin{aligned}
& f_{1}:=\frac{1}{12}\left(2 b_{1}+3 b_{2}+b_{3}\right), \quad f_{2}:=\frac{1}{3}\left(b_{1}-b_{3}\right), \\
& f_{3}:=\frac{1}{12}\left(2 b_{1}-3 b_{2}+b_{3}\right), \quad f_{4}:=-\frac{1}{2}\left(b_{1}+b_{2}-b_{4}-b_{5}\right), \\
& f_{5}:=-\frac{1}{2}\left(b_{1}-b_{2}-b_{4}+b_{5}\right), \quad f_{6}:=\frac{1}{12}\left(2 b_{1}-3 b_{4}+b_{6}\right), \\
& \bar{f}_{7}:=\frac{1}{24}\left(2 \bar{b}_{1}-\bar{b}_{3}-\bar{b}_{6}\right), \quad \bar{f}_{8}:=-\frac{1}{16}\left(\bar{b}_{1}+\bar{b}_{2}+\bar{b}_{4}+\bar{b}_{5}\right), \\
& \bar{f}_{9}:=-\frac{1}{16}\left(\bar{b}_{1}-\bar{b}_{2}-\bar{b}_{4}+\bar{b}_{5}\right), \quad \bar{f}_{10}:=-\frac{1}{8}\left(\bar{b}_{1}-\bar{b}_{5}\right) .
\end{aligned}
$$


Relying on the existence of three topological invariants (2.9), Karananas [25] imposed three conditions on the Lagrangian parameters in (B.1): $\bar{a}_{0}, f_{6}, \bar{f}_{8}=0$.

Based on (B.1), one can find a suitable form of the covariant momenta $\mathcal{H}_{i j k}$ and $\mathcal{H}_{i j k l}^{\prime}$ :

$$
\begin{aligned}
\mathcal{H}_{i j k}= & 4\left(h_{1} T_{i j k}+h_{2} T_{[k j] i}+h_{3} \eta_{i[j} V_{k]}\right), \\
& +4\left(\bar{h}_{4} \varepsilon_{j k}{ }^{m n} T_{i m n}+\bar{h}_{5} \varepsilon_{i[j}{ }^{m n} T_{m n k]}\right), \\
\mathcal{H}_{i j k l}^{\prime}= & 4\left[f_{1} R_{i j k l}+\frac{1}{2} f_{2}\left(R_{i[k j l]}-R_{j[k i l]}\right)+f_{3} R_{k l i j}\right] \\
& +2\left[f_{4}\left(\eta_{j[l} \hat{R}_{i k]}-\eta_{i[l} \hat{R}_{j k]}\right)+f_{5}\left(\eta_{j[l} \hat{R}_{k] i}-\eta_{i[l} \hat{R}_{k] j}\right)+f_{6}\left(\eta_{j l} \eta_{i k}-\eta_{i l} \eta_{j k}\right) R\right] \\
& +\bar{f}_{7}\left[2 \varepsilon_{i j k l} R+(\varepsilon R)\left(\eta_{i k} \eta_{j l}-\eta_{j k} \eta_{i l}\right)\right] \\
& +4\left(\bar{f}_{8} \varepsilon_{k l}{ }^{m n} R_{i j m n}+\bar{f}_{9} \varepsilon_{i j}{ }^{m n} R_{m n k l}\right)+2 \bar{f}_{10}\left(\varepsilon_{i j}{ }^{m n} R_{k l m n}+\varepsilon_{k l}{ }^{m n} R_{m n i j}\right) .
\end{aligned}
$$

\section{C $(3+1)$ decomposition of spacetime}

The dynamical content of canonical constraints is greatly clarified by using a decomposition of tensor fields with respect to the subgroup of $3 \mathrm{~d}$ rotations in the spatial hypersurface $\Sigma_{0}: x^{0}=$ const.

Let $\boldsymbol{e}_{\alpha}$ be a basis of three coordinate tangent vectors in $\Sigma_{0}, \boldsymbol{e}_{\alpha}=\partial_{\alpha}(\alpha=1,2,3)$, and $\boldsymbol{n}$ the unit normal to $\Sigma_{0}, n_{k}=h_{k}^{0} / \sqrt{g^{00}}$. The four vectors $\left(\boldsymbol{n}, \boldsymbol{e}_{\alpha}\right)$ define the so-called ADM basis of tangent vectors in spacetime. The decomposition of the vector $\boldsymbol{e}_{0}$ in the ADM basis is given by

$$
\boldsymbol{e}_{0}=N \boldsymbol{n}+N^{\alpha} \boldsymbol{e}_{\alpha}
$$

where $N$ and $N^{\alpha}$, known as the lapse and shift functions, respectively, are linear in $b_{0}^{k}$ :

$$
\begin{aligned}
& N=\boldsymbol{e}_{0} \boldsymbol{n}=n_{k} b_{0}^{k}=1 / \sqrt{g^{00}}, \\
& N^{\alpha}=\boldsymbol{e}_{0} \boldsymbol{e}_{\beta}{ }^{3} g^{\beta \alpha}=h_{\bar{k}}^{\alpha} b^{k}{ }_{0}=-g^{0 \alpha} / g^{00} .
\end{aligned}
$$

Introducing the projectors on $\boldsymbol{n}$ and $\boldsymbol{e}_{\alpha}$, given by $\left(P_{\perp}\right)_{k}^{l}=n_{k} n^{l}$ and $\left(P_{\|}\right)_{k}^{l}=\delta_{k}^{l}-n_{k} n^{l}$, respectively, one can express a spacetime vector $V_{k}$ in terms of its orthogonal (to $\left.\Sigma_{0}\right)$ and "parallel" (living in the tangent space of $\Sigma_{0}$ ) components:

$$
V_{k}=n_{k} V_{\perp}+V_{\bar{k}}
$$

where $V_{\perp}:=n^{k} V_{k}$ and $V_{\bar{k}}:=V_{k}-n_{k} V_{\perp}$ Here, we use a convention that a bar over an index ' $\mathrm{k}$ ' denotes its parallel projection, so that $n^{k} V_{\bar{k}}$ vanishes. The objects $V_{\perp}$ and $V_{\bar{k}}$ are respectively a scalar and a vector with respect to $3 \mathrm{~d}$ rotations in $\Sigma_{0}$.

Consider now a second-rank tensor $X_{i k}$. Its orthogonal-parallel decomposition reads

$$
X_{i k}=n_{i} X_{\perp \bar{k}}+n_{i} n_{k} X_{\perp \perp}+n_{k} X_{\bar{\imath} \perp}+X_{\bar{\imath} \bar{k}}
$$

Here, $X_{\perp \bar{k}}$ is a vector and $X_{\perp \perp}$ a scalar with respect to 3 d rotations, whereas the irreducible parts of $X_{\bar{\imath} \bar{k}}$ are its trace, antisymmetric and traceless symmetric parts:

$$
\begin{aligned}
& { }^{S} X:=X^{\bar{k}}{ }_{\bar{k}}, \quad{ }^{A} X_{\bar{\imath} \bar{k}}:=X_{[\bar{\imath} \bar{k}]}, \quad{ }^{T} X_{\bar{\imath} \bar{k}}:=X_{(\bar{\imath} \bar{k})}-\frac{1}{3} \eta_{\bar{\imath} \bar{k}} X^{\bar{m}}{ }_{\bar{m}}, \\
& X_{\bar{\imath} \bar{k}}={ }^{A} X_{\bar{\imath} \bar{k}}+{ }^{T} X_{\bar{\imath} \bar{k}}+\frac{1}{3} \eta_{\bar{\imath} \bar{k}}{ }^{S} X .
\end{aligned}
$$


As a consequence,

$$
X^{\bar{\imath} \bar{k}} Y_{\bar{\imath} \bar{k}}={ }^{A} X_{\bar{\imath} \bar{k}}{ }^{A} Y_{\bar{\imath} \bar{k}}+{ }^{T} X_{\bar{\imath} \bar{k}}{ }^{T} Y_{\bar{\imath} \bar{k}}+\frac{1}{3}{ }^{S} X^{S} Y
$$

Now, it is straightforward to extend these considerations to any tensor. As a particularly interesting example, we consider the spacetime tensor $X_{i j k}=-X_{j i k}$, which is decomposed into the set of spatial tensors $\left(X_{\perp \bar{\jmath} \perp}, X_{\perp \bar{\jmath} \bar{k}}, X_{\bar{\imath} \bar{\jmath} \perp}, X_{\bar{\imath} \bar{\jmath} \bar{k}}\right)$. The irreducible parts of $X_{\bar{\imath} \bar{\jmath} \bar{k}}=-X_{\bar{\jmath} \bar{k}}$ are the pseudoscalar, the vector and the traceless symmetric tensor, respectively:

$$
\begin{aligned}
& { }^{P} X:=\varepsilon^{\bar{\imath} \bar{\jmath} \bar{k}} X_{\bar{\imath} \bar{\jmath} \bar{k}}, \quad{ }^{V} X_{\bar{\imath}}:=X_{\bar{\imath} \bar{k}}{ }^{\bar{k}}, \\
& { }^{T} X_{\bar{\imath} \bar{\jmath} \bar{k}}:=X_{\bar{\imath}(\bar{\jmath} \bar{k})}+\frac{1}{2} \eta_{\bar{\imath}(\bar{\jmath}}{ }^{V} X_{\bar{k})}-\frac{1}{2} \eta_{\bar{\jmath} \bar{k}}{ }^{V} X_{\bar{\imath}} .
\end{aligned}
$$

The tensor part satisfies the cyclic identity ${ }^{T} X_{\bar{\imath} \bar{\jmath} \bar{k}}+{ }^{T} X_{\bar{k} \bar{\imath} \bar{\jmath}}+{ }^{T} X_{\bar{\jmath} \bar{k} \bar{\imath}}=0$. The epsilon tensor $\varepsilon_{\bar{\imath} \bar{\jmath} \bar{k}}$ is defined by $\varepsilon_{\bar{\imath} \bar{\jmath} \bar{k}}:=\varepsilon_{\perp \bar{\jmath} \bar{\jmath} \bar{k}}$ and satisfies the identities

$$
\begin{aligned}
& \varepsilon_{\bar{\imath} \bar{\jmath} \bar{k}} \varepsilon^{\bar{m} \bar{n} \bar{k}}=-\left(\delta_{\bar{\imath}}^{\bar{m}} \delta_{\bar{\jmath}}^{\bar{n}}-\delta_{\bar{\jmath}}^{\bar{m}} \delta_{\bar{\imath}}^{\bar{n}}\right), \\
& \varepsilon_{\bar{\imath} \bar{n} \bar{k}} \varepsilon^{\bar{m} \bar{n} \bar{k}}=-3 \delta_{\bar{\imath}}^{\bar{m}}, \quad \varepsilon_{\bar{m} \bar{n} \bar{k}} \varepsilon^{\bar{m} \bar{n} \bar{k}}=-6 .
\end{aligned}
$$

The related decomposition formulas read:

$$
\begin{aligned}
& X_{\bar{\imath} \bar{\jmath} \bar{k}}=\frac{4}{3}{ }^{T} X_{[\bar{\imath}] \bar{j}}-\eta_{\bar{k}[\bar{\imath}}{ }^{V} X_{\bar{\jmath}]}-\frac{1}{6} \varepsilon_{\bar{\imath} \bar{\jmath} \bar{k}}{ }^{P} X, \\
& X^{\bar{\imath} \bar{\jmath} \bar{k}} Y_{\bar{\imath} \bar{\jmath} \bar{k}}=\frac{4}{3}^{T} X^{\bar{\imath} \bar{\jmath} \bar{k}}{ }^{T} Y_{\bar{\imath} \bar{\jmath} \bar{k}}+{ }^{V} X^{\bar{\imath}}{ }^{V} Y_{\bar{\imath}}-\frac{1}{6}{ }^{P} X^{P} Y .
\end{aligned}
$$

\section{General construction of $\mathcal{H}_{\perp}$}

In this appendix, we discuss the general structure of $\mathcal{H}_{\perp}$, including the case when some of the critical parameters vanish. In a simplified but self-evident notation, the relations that define critical parameters have the following typical form (see Sec. 3):

$$
\varphi=F V
$$

where

$$
\varphi:=\left(\begin{array}{c}
\varphi_{1} \\
\varphi_{2}
\end{array}\right), \quad F:=\left(\begin{array}{cc}
a & \bar{b} \\
\bar{c} & d
\end{array}\right), \quad V:=\left(\begin{array}{c}
V_{1} \\
V_{2}
\end{array}\right) .
$$

Here, $\varphi$ represents the if-constraints, $V$ are the corresponding velocities, and $F$ is the matrix with eigenvalues $c_{1}, c_{2}$. Since $F$ is chosen to represent $A, B_{0}, B_{1}$ or $B_{2}$, the parameter $\bar{c}$ is proportional to $\bar{b}, \bar{c}=\kappa \bar{b}$. If $\bar{b}=0$, the matrix $F$ is already diagonal, and the construction of $\mathcal{H}_{\perp}$ is quite simple. When $\bar{b} \neq 0$, which is typical for the parity-violating PG, the matrix $F$ needs first to be diagonalized. The diagonal form $D$ of $F$ is constructed as

$$
D=P^{-1} F P, \quad P:=\left(\begin{array}{cc}
-\bar{b} & -\bar{b} \\
a-c_{1} & a-c_{2}
\end{array}\right), \quad D=\left(\begin{array}{cc}
c_{1} & 0 \\
0 & c_{2}
\end{array}\right),
$$

where $P$ is invertible provided $\operatorname{det} P=\bar{b}\left(c_{2}-c_{1}\right) \neq 0$, and

$$
P^{-1}=\frac{1}{\operatorname{det} P}\left(\begin{array}{cc}
a-c_{2} & \bar{b} \\
-a+c_{1} & -\bar{b}
\end{array}\right) \text {. }
$$


Left multiplication of (D.1) by $P^{-1}$ yields

$$
\varphi^{\prime}=D V^{\prime}
$$

where $\varphi^{\prime}:=P^{-1} \varphi$ and $V^{\prime}:=P^{-1} V$, or equivalently,

$$
\varphi_{1}^{\prime}=c_{1} V_{1}^{\prime}, \quad \varphi_{2}^{\prime}=c_{2} V_{2}^{\prime} .
$$

To construct the related $F$-part of $\mathcal{H}_{\perp}$, note that its typical form reads

$$
\mathcal{H}_{\perp}^{F}=\varphi^{T} Q V \equiv \varphi^{T}\left(P^{T} Q P\right) V^{\prime}, \quad Q:=\left(\begin{array}{cc}
q_{1} & 0 \\
0 & q_{2}
\end{array}\right)
$$

see Sec. 4. Further discussion depends on the specific values of $c_{1}$ and $c_{2}$.

(1) When $c_{1}, c_{2} \neq 0$, Eq. (D.1) implies $V=F^{-1} \varphi$, and $\mathcal{H}_{\perp}^{F}=\varphi^{T} Q F^{-1} \varphi$ coincides with the result found in Sec. 4. (2) The case $c_{1}=c_{2}=0$ is rather trivial: both if-constraints $\varphi_{n}^{\prime}$ become true constraints that appear in the total Hamiltonian, but $\mathcal{H}_{\perp}^{F}=0$. (3) Finally, when only one critical parameter vanishes (which requires $\operatorname{det} F=0$ ), say $c_{2}=0$, then

$\varphi_{2}^{\prime}=0$ (a new constraint), $V_{2}^{\prime}$ remains undetermined and $\varphi_{1}^{\prime}=c_{1} V_{1}^{\prime}$. Hence, (D.4 implies that

$$
\mathcal{H}_{\perp}^{F}=\left(\bar{b}^{2} q_{1}+d^{2} q_{2}\right) \frac{1}{c_{1}}\left(\varphi_{1}^{\prime}\right)^{2}+\varphi_{1}^{\prime}\left(\bar{b}^{2} q_{1}-a d q_{2}\right) V_{2}^{\prime} .
$$

The result can be also expressed in terms of the original if-constraints $\varphi_{n}$ by noting that $\varphi_{2}^{\prime}=$ 0 implies $\varphi_{1}^{\prime}=-\varphi_{1} / \bar{b}$. The factor $1 / c_{1}$ in the first term shows a typical dependence on the critical parameters, known from $\mathrm{PG}^{+}$, whereas the second term, linear in the undetermined velocity $V_{2}^{\prime}$, can be absorbed into the total Hamiltonian, see [14, 15, 23]. The presence of an extra constraint $\varphi_{2}^{\prime}$ requires to complete the whole consistency procedure.

In the context of the weak field approximation, the form of $\mathcal{H}_{\perp}^{F}$ in (D.5) determines the no-ghost conditions for the case (3):

$$
\operatorname{det} F=a d-\bar{b} \bar{c}=0, \quad \sigma c_{1}>0,
$$

where $\sigma$ is the sign of $\left(\bar{b}^{2} q_{1}+d^{2} q_{2}\right)$ and $c_{1}=a+d$.

Now, we have a comment on kind of "non-analiticity" of the above results. Since the assumption $\bar{b} \neq 0$ ensures the regularity of the matrix $P$, the diagonal matrix $D$ in (D.2) has no valid limit for $\bar{b} \rightarrow 0$. Hence, the expressions for $c_{n}$ when $\bar{b}=0$ cannot be obtained by taking the limit $\bar{b} \rightarrow 0$ of the generic result. However, since the matrix $F$ for $\bar{b}=0$ is already diagonal, the critical parameters $c_{n}$ can be obtained directly from $F$. The same conclusion also holds for the form of $\mathcal{H}_{\perp}^{F}$.

\section{E Linearized Bianchi identities}

In Secs. 5] and 6, many technical simplifications were obtained with the help of the linearized Bianchi identities,

$$
\varepsilon^{\mu \nu \lambda \rho} R^{i j}{ }_{\nu \lambda \rho}=0, \quad \varepsilon^{\mu \nu \lambda \rho} \partial_{\nu} T_{i \lambda \rho}=\varepsilon^{\mu \nu \lambda \rho} R_{i \nu \lambda \rho},
$$


and their consequences. In particular, the first identity implies that

$$
\partial^{k} X_{i k}=0, \quad \partial^{i} G_{i k}=0,
$$

where $G_{i k}:=R i c_{i k}-(1 / 2) \eta_{i k} R$, and the second identity yields

$$
\begin{aligned}
& X_{i}^{j}=-\frac{1}{2} \varepsilon^{j k m n} \partial_{k} T_{i m n}, \quad X=3 \partial \mathcal{A}, \\
& \varepsilon^{i j m n} R_{i j m k}=2 X_{k}{ }^{n}-\delta_{k}^{n} X, \\
& 2 R i c_{[m n]}=-\partial^{k} T_{k m n}+2 \partial_{[m} \mathcal{V}_{n]} .
\end{aligned}
$$

As a consequence,

$$
\partial^{m} \Phi_{i m}=\partial^{m} \hat{R}_{[i m]}+\frac{1}{4} \partial_{i} R, \quad \partial^{m} \Psi_{i m}=\partial^{m} X_{[m i]}-\frac{1}{4} \partial_{i} X .
$$

\section{F Simplified conditions for the absence of tachyons}

In this appendix, we derive a simplified form of the conditions (7.9) and (7.16), describing the absence of tachyons in the spin- 0 and spin- 1 sectors, respectively; the spin-2 sector is discussed in subsection 7.3 ,

\section{Spin-0 sector}

The expression for $\operatorname{tr} f_{0}$, found in subsection 6.1, can be represented in a suitable form as

$$
\frac{1}{3} \operatorname{tr} f_{0}=\alpha_{0}\left(b_{2}+b_{3}\right)+\beta_{0}\left(\bar{b}_{2}-\bar{b}_{3}\right)+\gamma_{0}\left(b_{4}+b_{6}\right),
$$

where

$$
\begin{aligned}
& \alpha_{0}=a_{0}\left(2 a_{0}+a_{2}\right), \quad \beta_{0}=4 a_{0}\left(\bar{a}_{2}-\bar{a}_{0}\right), \\
& \gamma_{0}=-\left[a_{2}\left(a_{0}+2 a_{3}\right)+2\left(\bar{a}_{0}-\bar{a}_{2}\right)^{2}\right] .
\end{aligned}
$$

After dividing this equation by $\left(b_{4}+b_{6}\right)>0$, one obtains

$$
\frac{\operatorname{tr} f_{0}}{3\left(b_{4}+b_{6}\right)}=\alpha_{0} \frac{b_{2}+b_{3}}{b_{4}+b_{6}}+\beta_{0} \frac{\bar{b}_{2}-\bar{b}_{3}}{b_{4}+b_{6}}+\gamma_{0} .
$$

By noting that the first relation in (7.6) can be written as

$$
\frac{b_{2}+b_{3}}{b_{4}+b_{6}}+x^{2}<0, \quad x:=\frac{\bar{b}_{2}-\bar{b}_{3}}{b_{4}+b_{6}},
$$

we find it useful to rewrite (F.1a) in the form

$$
\begin{aligned}
& \frac{\operatorname{tr} f_{0}}{3\left(b_{4}+b_{6}\right)}=\alpha_{0}\left(\frac{b_{2}+b_{3}}{b_{4}+b_{6}}+x^{2}\right)+F_{0}(x), \\
& F_{0}(x):=-\alpha_{0} x^{2}+\beta_{0} x+\gamma_{0} .
\end{aligned}
$$


Further analysis is based on an important property of the quadratic function $F_{0}(x)$, based on (7.9a): its discriminant, $\Delta_{0}=\beta_{0}^{2}+4 \alpha_{0} \gamma_{0}$, is always negative,

$$
\Delta_{0}=-4 a_{0} a_{2}\left[\left(2 a_{0}+a_{2}\right)\left(a_{0}+2 a_{3}\right)+2\left(\bar{a}_{0}-\bar{a}_{2}\right)^{2}\right] \equiv(4 / 3) a_{0} a_{2} \operatorname{det} N_{0}<0 .
$$

Similar considerations applied to $a_{2} \operatorname{tr} f_{0}$ modify Eq. (F.1b) by an overall multiplicative factor $a_{2}$. To simplify the discussion, we introduce a suitable notation: $\alpha_{0}^{\prime}:=a_{2} \alpha_{0}$ and $F_{0}^{\prime}(x):=a_{2} F_{0}(x)$. Note that the discriminant $\Delta_{0}^{\prime}$ of the new function $F_{0}^{\prime}(x)$ remains negative. Now, we are ready to prove the following statement:

S0. Given $\Delta_{0}<0$, the condition $\alpha_{0}^{\prime} \equiv a_{2} \alpha_{0}>0$ is equivalent to $a_{2} \operatorname{tr} f_{0}<0$.

To prove this equivalence, we start by assuming $\alpha_{0}^{\prime}>0$, which implies

$$
a_{2} \operatorname{tr} f_{0}<3\left(b_{4}+b_{6}\right) F_{0}^{\prime}(x) .
$$

Moreover, the parabola $F_{0}^{\prime}(x)$ opens downward, and $\Delta_{0}^{\prime} / \alpha_{0}^{\prime}<0$ (negative at vertex) ensures that $F_{0}^{\prime}(x)<0$ for any $x$. Hence, $a_{2} \operatorname{tr} f_{0}<0$, what was to be shown.

The reverse statement $a_{2} \operatorname{tr} f_{0}<0 \Rightarrow \alpha_{0}^{\prime}>0$ can be easily proven by reductio ad absurdum, that is by showing that $\alpha_{0}^{\prime}<0$ implies $a_{2} \operatorname{tr} f_{0}>0$, which is a contradiction.

The statement $\mathbf{S} \mathbf{0}$ allows us to replace (7.9b) with the much simpler condition $a_{2}>0$.

\section{Spin-1 sector}

For the spin-1 sector, we first rewrite $\operatorname{tr} f_{1}$ in the form

$$
\frac{1}{4} \operatorname{tr} f_{1}=\alpha_{1}\left(b_{4}+b_{5}\right)+\beta_{1}\left(\bar{b}_{2}-\bar{b}_{5}\right)+\gamma_{1}\left(b_{2}+b_{5}\right),
$$

where

$$
\begin{aligned}
\alpha_{1} & :=2 \mu_{3} k_{2} A_{0}-4 \mu_{3} \bar{A}_{0}^{2}+2 A_{0}\left(\bar{a}_{0}-\bar{a}_{2}\right)^{2}, \\
\beta_{1} & :=-4 \mu_{2} \mu_{3} \bar{A}_{0}+4\left(A_{0}^{2}+\bar{A}_{0}^{2}\right)\left(\bar{a}_{0}-\bar{a}_{2}\right)-4 \bar{A}_{0}\left(\bar{a}_{0}-\bar{a}_{2}\right)^{2}, \\
\gamma_{1} & :=2 A_{0} \mu_{2} k_{3}-\mu_{2} \bar{A}_{0}^{2}+2 A_{0}\left(\bar{a}_{0}-\bar{a}_{2}\right)^{2} .
\end{aligned}
$$

After dividing by $\left(b_{2}+b_{5}\right)>0$, one can rewrite $(\mathrm{F} .4 \mathrm{a})$ in a suitable form

$$
\begin{aligned}
& \frac{1}{4\left(b_{2}+b_{5}\right)} \operatorname{tr} f_{1}=\alpha_{1}\left(\frac{b_{4}+b_{5}}{b_{2}+b_{5}}+x^{2}\right)+F_{1}(x), \\
& F_{1}(x):=-\alpha_{1} x^{2}+\beta_{1} x+\gamma_{1}, \quad x:=\frac{\bar{b}_{2}-\bar{b}_{5}}{b_{2}+b_{5}},
\end{aligned}
$$

As a consequence of (7.16a), the discriminant $\Delta_{1}$ of the quadratic function $F_{1}(x)$ is automatically negative,

$$
\begin{aligned}
\Delta_{1}:= & 16\left(A_{0}^{2}+\bar{A}_{0}^{2}\right)\left[\mu_{2} \mu_{3}+\left(\bar{a}_{0}-\bar{a}_{2}\right)^{2}\right]\left[k_{2} k_{3}+\left(\bar{a}_{1}-\bar{a}_{2}\right)^{2}\right] \\
\equiv & \frac{2}{3}\left(A_{0}^{2}+\bar{A}_{0}^{2}\right)\left(\operatorname{det} N_{1}\right)(\operatorname{det} A)<0 .
\end{aligned}
$$

To relate our considerations to the properties of $(\operatorname{det} A) \operatorname{tr} f_{1}$, we multiply Eq. ( $(\underline{\mathrm{F} .4 \mathrm{~b}})$ by $\operatorname{det} A$, and introduce a suitable notation $\alpha_{1}^{\prime}:=(\operatorname{det} A) \alpha_{1}$ and $F_{1}^{\prime}(x):=(\operatorname{det} A) F_{1}(x)$. The new discriminant $\Delta_{1}^{\prime}$ is also negative. Now, one can prove the following statement: 
S1. For $\Delta_{1}<0$, the condition $\alpha_{1}^{\prime} \equiv(\operatorname{det} A) \alpha_{1}<0$ is equivalent to $(\operatorname{det} A) \operatorname{tr} f_{1}>0$.

The proof goes as follows. Starting with $\alpha_{1}^{\prime}<0$, one obtains

$$
(\operatorname{det} A) \operatorname{tr} f_{1}>4\left(b_{2}+b_{5}\right) F_{1}^{\prime}(x) .
$$

Then, by noting that the parabola opens upward $\left(\alpha_{1}^{\prime}<0\right)$ and $\Delta_{1}^{\prime} / \alpha_{1}^{\prime}>0$ (positive at vertex), one concludes that $F_{1}^{\prime}(x)>0$. Hence, $(\operatorname{det} A) \operatorname{tr} f_{1}>0$.

As before, the reverse statement $(\operatorname{det} A) \operatorname{tr} f_{1}>0 \Rightarrow \alpha_{1}^{\prime}<0$ can be proven by showing that $\alpha_{1}^{\prime}>0$ leads to $(\operatorname{det} A) \operatorname{tr} f_{1}<0$, which is a contradiction.

The condition $\Delta_{1}<0$, combined with $(\operatorname{det} A) \alpha_{1}<0$, can be realized in two ways:

$$
\begin{aligned}
& \text { (i) } \quad \operatorname{det} A>0, \quad \operatorname{det} N_{1}<0, \quad \alpha_{1}<0 \text {. } \\
& \text { (ii) } \quad \operatorname{det} A<0, \quad \operatorname{det} N_{1}>0, \quad \alpha_{1}>0 \text {. }
\end{aligned}
$$

\section{References}

[1] H. Weyl, Electron and gravitation, I, Zeitschrift für Physik, 56 (1929) 330-352 (in German); English translation in: L. O'Raifeartaigh, The Dawning of Gauge Theory (Princeton University Press, Princeton, 1997), pp. 121-144.

[2] C. N. Yang and R. Mills, Conservation of isotopic spin and isotopic gauge invariance, Phys. Rev. 96, 191-195 (1954);

R. Utiyama, Invariant theoretical interpretation of interactions, Phys. Rev. 101, 15971607 (1956).

[3] T. W. B. Kibble, Lorentz invariance and the gravitational field, J. Math. Phys. 2, 212-221 (1961);

D. W. Sciama, The analogy between charge and spin in general relativity, in: Recent Developments in General Relativity, Festschrift for Infeld (Pergamon Press, Oxford; PWN, Warsaw, 1962) pp. 415-439.

[4] F. W. Hehl, Four lectures on Poincaré gauge theory, in: Proceedings of the 6th Course of the School of Cosmology and Gravitation on Spin, Torsion, Rotation and Supergravity, Erice, Italy, 1979, eds. P. G. Bergmann, V. de Sabbata (Plenum, New York, 1980) pages 5-61.

[5] K. Hayashi and T. Shirafuji, Gravity from Poincaré gauge theory of fundamental interactions. I General formulation, Prog. Theor. Phys. 64, 866-882 (1980); II Equations of motion for test bodies and various limits, Prog. Theor. Phys. 64, 883-896 (1980); III Weak field approximation, Prog. Theor. Phys. 64, 1435-1452 (1980); IV Mass and energy of particle spectrum, Prog. Theor. Phys. 64, 2222-2241 (1980).

[6] F. W. Hehl, J. D. McCrea, E. W. Mielke, and Y. Ne'eman, Metric-affine gauge theory of gravity: feld equations, Noether identities, world spinors, and breaking of dilation invariance, Phys. Rep. 258, 1-177 (1995). 
[7] M. Blagojević, Gravitation and Gauge Symmetries (IoP, Bristol, 2002).

[8] M. Blagojević and F. W. Hehl (eds.), Gauge Theories of Gravitation, A Reader with Commentaries (Imperial College Press, London, 2013).

[9] V. N. Ponomariov, A. O. Barvinsky and Yu. N. Obukhov, Gauge Approach and Quantization Methods in Gravity Theory (Nauka, Moscow, 2017). This book offers an impressive list of 3136 references on gauge theories of gravity.

[10] E. W. Mielke, Geometrodynamics of Gauge Fields, 2nd. edition (Springer International, Basel, 2017).

[11] Yu. N. Obukhov, Poincaré gauge gravity: Selected topics, Int. J. Geom. Meth. Mod. Phys. 03, 95-138 (2006).

[12] E. Sezgin and P. van Nieuwenhuizen, New ghost-free gravity Lagrangians with propagating torsion, Phys. Rev. 21, 3269-3280 (1980);

E. Sezgin, Class of ghost-free gravity Lagrangians with massive or massless propagating modes, Phys. Rev. 24, 1677-1680 (1981).

[13] P. A. M. Dirac, Lectures on Quantum Mechanics (New York, Yeshiva University, 1964);

A. Hanson, T. Regge and C. Teitelboim, Constrained Hamiltonian Dynamics (Academia Nacionale dei Lincei, Rome, 1976);

K. Sundermeyer, Constrained Dynamics (Springer, Berlin, 1982);

M. Henneaux and C. Teitelboim, Quantization of gauge systems (Princeton University Press, Princeton, 1992).

[14] M. Blagojević and I. Nikolić, Hamiltonian dynamics of Poincaré gauge theory: General structure in the time gauge, Phys. Rev. D 28, 2455-2463 (1983).

[15] I. Nikolić, Dirac Hamiltonian structure of $R+R^{2}+T^{2}$ Poincaré gauge theory of gravity without gauge fixing, Phys. Rev. D 30, 2508-2520 (1984).

[16] W.-H. Cheng, D.-C. Chern, and J. M. Nester, Canonical analysis of the one-parameter teleparallel theory, Phys. Rev. D 38, 2656-2658 (1988).

[17] H. Chen, J. M. Nester, and H.-J. Yo, Acausal PGT modes and the nonlinear constraint effect, Acta Physica Polonica B 29, 961-970 (1998).

[18] H.-J. Yo and J. M. Nester, Hamiltonian analysis of Poincaré gauge theory scalar modes, Int. J. Mod. Phys. D 08, 459-479 (1999); Hamiltonian analysis of Poincaré gauge theory: Higher spin modes, Int. J. Mod. Phys. D 11, 747-780 (2002).

[19] K.-F. Shie, J. M. Nester, and H.-J. Yo, Torsion cosmology and the accelerating universe, Phys. Rev. D 78, 023522 (2008) (16 pages). 
[20] R. Hojman, C. Mukku, and W. A. Sayed, Parity violation in metric torsion theories of gravitation, Phys. Rev. D 22, 1915-1921 (1980);

Yu. N. Obukhov, V. N. Ponomarev and V. V. Zhytnikov, Quadratic Poincaré gauge theory of gravity: a comparison with the general relativity theory, Gen. Rel. Grav. 21, 1107-1142 (1989).

[21] P. Baekler, F. W. Hehl, and J. M. Nester, Poincaré gauge theory of gravity: Friedman cosmology with even and odd parity modes: Analytic part, Phys. Rev. D 83, 024001 (2011) (23 pages);

P. Baekler, and F. W. Hehl, Beyond Einstein-Cartan gravity: quadratic torsion and curvature invariants with even and odd parity including all boundary terms, Class. Quantum Grav. 28, 215017 (2011) (11 pages);

[22] D. Diakonov, A. G. Tumanov, and A. A. Vladimirov, Low-energy general relativity with torsion: A systematic derivative expansion, Phys. Rev. D 84, 124042 (2011) (16 pages).

[23] F.-H. Ho and J. M. Nester, Poincaré gauge theory with even and odd parity dynamic connection modes: isotropic Bianchi cosmological models, J. Phys. Conf. Ser. 330, 012005 (2011) (8 pages); Poincaré gauge theory with coupled even and odd parity dynamic spin-0 modes: dynamical equations for isotropic Bianchi cosmologies, Int. J. Mod. Phys. D 20, 2125-2138 (2011);

F.-H. Ho, H. Chen, J. M. Nester, and H.-J. Yo, General Poincaré gauge theory cosmology, Chin. J. Phys. 53, 110109 (2015) (21 pages).

[24] Yu. N. Obukhov and F. W. Hehl, Extended Einstein-Cartan theory a la Diakonov: the field equations, Phys. Lett. B 713 321-325 (2012).

[25] G. K. Karananas, The particle spectrum of parity-violating Poincaré gravitational theory, Class. Quantum Grav. 32, 055012 (2015) (38 pages); Corrigendum: Class. Quantum Grav. 32, 089501 (2015) (2 pages).

[26] Yu. N. Obukhov, Gravitational waves in Poincaré gauge gravity theory, Phys. Rev. D 95, 084028 (2017) (12 pages).

[27] M. Blagojević, B. Cvetković and Yu. N. Obukhov, Generalized plane waves in Poincaré gauge theory of gravity, Phys. Rev. D 96, 064031 (2017) (13 pages).

[28] M. Blagojević and M. Vasilić, Phys. Rev. D 36 1679-1684 (1987).

[29] M. Blagojević and B. Cvetković, Generalized pp waves in Poincaré gauge theory, Phys. Rev. D 95, 104018 (2017) (10 pages).

[30] Calculations performed in this paper are checked using the Excalc package of the computer algebra system Reduce, and Wolfram Mathematica. 